\title{
Biomechanically Constrained Groupwise Statistical Shape Model to Ultrasound Registration of the Lumbar Spine
}

\author{
by
}

\section{SiAVASH KHALLAGHI}

\author{
A thesis submitted to the \\ Department of Electrical and Computer Engineering \\ in conformity with the requirements for \\ the degree of Master of Applied Science
}

Queen's University

Kingston, Ontario, Canada

September 2010

Copyright (c) Siavash Khallaghi, 2010 


\section{Abstract}

Spinal needle injections for back pain management are frequently carried out in hospitals and radiological clinics. Currently, these procedures are performed under fluoroscopy or CT guidance in specialized interventional radiology facilities. As an alternative, the use of inexpensive ultrasound image guidance promises to improve the efficacy and safety of these procedures. We propose to eliminate or reduce the need for ionizing radiation, by creating and registering a statistical shape model of the lumbar vertebrae to 3D ultrasound volumes of patient, using a groupwise registration algorithm. From a total of 35 patient CT volumes, a statistical shape model of the L2, L3 and L4 vertebrae is built, including the mean shape, and principal modes of variation. The statistical shape model is registered to the $3 \mathrm{D}$ ultrasound by interchangeably optimizing the model parameters and their relative poses. We also use a biomechanical model to constrain the relative motion of the models throughout the registration process. Validation is performed on three tissue mimicking-phantoms designed to preserve realistic curvature of the spine. We compare pairwise and groupwise registration of the statistical shape model of the spine and demonstrate that clinically acceptable mean target error registration of $2.4 \mathrm{~mm}$ can be achieved with the proposed method. Registration results also show that the groupwise registration outperforms the pairwise in terms of success rate. 


\section{Acknowledgments}

I would first like to thank my supervisors, Drs. Purang Abolmaesumi and Parvin Mousavi, for their guidance, support and enthusiasm. I would also like to thank Dr. Gabor Fichtinger for his encouragement and generous advice, especially in times when things where not looking well. I would especially like to thank Dr. Dan Borscneck, for helping me with my experiments and helpful discussions. This work would have never been possible without their encouragement and motivation that kept me working hard throughout my post graduate studies.

I would also like to thank all my friends and colleagues here at Queen's, especially those in the MedIA, Med-i and Perk labs: Siddharta Vikal, Pascal Fallavollita, Reza Seifabadi, Ehsan Dehghan, Hadi Tadayyon, Amir Tahmasebi, Ren Hui Gong, Sean Gill, Andrew Lang, Andrew Dickinson, Sahar Ghanavati, Tamas Ungi, Andras Lasso, Paweena U-Thainual, Yashar Madjidi, Alexandra Pompeu-Robinson, Hamed Peikari, Mohammad Peikari and all of you whom I have grown to know well in the past two years. Also to Ehsan, Yashar, Tamas, Hossein and Hadi: Thank you for bringing joy and friendship to the working environment and for being a "friend" in the absolute meaning. Thank you to all post-doctoral follows and researchers for being open to discussions and ready to lend a helping hand, especially Siddharta Vikal, Pascal Fallavollita and Andras Lasso. A big thanks to everyone in the administrative and 
technical staff in the ECE department and the School of Computing, especially Debra Frasier and Bernice Ison. It has been a pleasure knowing everyone, and I would have never traded the time that I spent here with anything else.

I would also like to thank my parents, Dr. Ali Khallaghi and Leili Riahi. I cannot put down in words all the sacrifices that you made throughout the years and without your love and support, I would never have had the fortune of being where I am today. Finally, I would like to thank Elmira Mohiyedin Bonab, for the love that we shared together.

"And there came out of the sanctuary a woman whose name was Almitra. And she was a seeress. And he looked upon her with exceeding tenderness, for it was she who had first sought and believed in him when he had been but a day in their city. Then said Almitra: "Speak to us of Love." " The Prophet, Gibran Khalil Gibran 


\section{Table of Contents}

Abstract $\quad$ i

$\begin{array}{ll}\text { Acknowledgments } & \text { ii }\end{array}$

Table of Contents $\quad$ iv

List of Tables $\quad$ vi

List of Figures $\quad$ vii

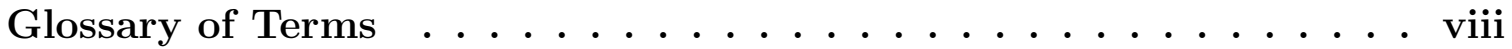

Statement of Co-authorship . . . . . . . . . . . $\quad$ x

Chapter 1:

Introduction .................. 1

1.1 Motivation . . . . . . . . . . . . . . . . . . 1

1.2 Proposed Image Guided Surgical System . . . . . . . . . . . . . . . . 6

1.3 Thesis Objectives . . . . . . . . . . . . . . . . . 7

1.4 Contributions . . . . . . . . . . . . . . . . . 8

1.5 Organization of Thesis .................... 9

Chapter 2:

Background ................... 11

2.1 Anatomy of the Lumbar Vertebrae . . . . . . . . . . . . . 11

2.2 Statistical Shape Models . . . . . . . . . . . . . . . . . . . . . . 12

2.3 Multi Modal Image Registration . . . . . . . . . . . . . . . . . . . . . . . 15

2.4 GPU-based Acceleration . . . . . . . . . . . . . . . . 17

Chapter 3:

Registration of a SSM of the Lumbar Spine... . . . . . 20

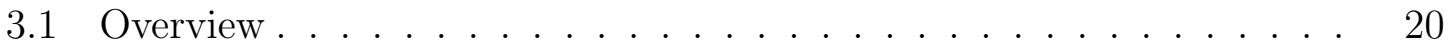




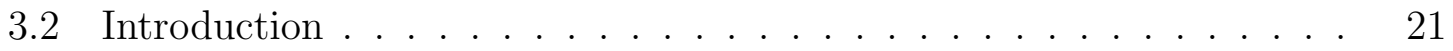

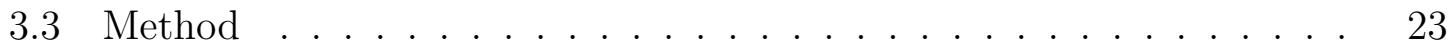

3.4 Experiments and Results . . . . . . . . . . . . . . . . . 26

Chapter 4:

Biomechanically Constrained Groupwise SSM... . . . . . 31

4.1 Overview . . . . . . . . . . . . . . . . . . . . . 31

4.2 Introduction . . . . . . . . . . . . . . . . . . . 32

4.3 Methods . . . . . . . . . . . . . . . . . . . . 35

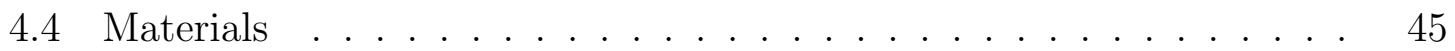

4.5 Experiments and Results . . . . . . . . . . . . . . . . . . 47

4.6 Discussion . . . . . . . . . . . . . . . . . . . . . . . 53

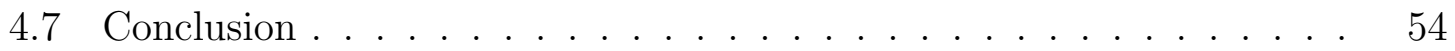

\section{Chapter 5:}

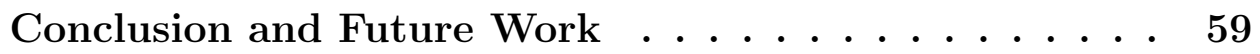

5.1 Summary of Contributions . . . . . . . . . . . . . . . . . . 59

5.2 Future Work . . . . . . . . . . . . . . . . . . . 61

Bibliography ........................ 63

Appendix A:

Parallel Implementation on the GPU $\ldots \ldots \ldots \ldots$ 


\section{List of Tables}

3.1 Registration results for the atlas to US registration. SR (Success Rate) is defined as the ratio of the registrations where the overall TRE is less than $3.5 \mathrm{~mm}$. SR is presented for each phantom with the maximum initial misalignment of $10 \mathrm{~mm}$. . . . . . . . . . . . . . . .

4.1 CPU vs. GPU Instantiation run-times and the performance gain computed as CPU/GPU. A total of 100 speed tests are performed on the CPU and the GPU. In each test, a random combination of SSM weights is passed to the instantiation block. . . . . . . . . . . . . . . . 44

4.2 Recipe for the phantom construction in Figure 4.6 . . . . . . . . . . 47

4.3 Results for the leave-one-out experiments for each vertebrae . . . . . 48

4.4 Results for the SSM to ultrasound pairwise registration. SR (Success Rate) is defined as the ratio of the registrations where the overall TRE is less than $3.0 \mathrm{~mm}$. SR is presented for each phantom with the maximum initial misalignment of $10 \mathrm{~mm}$. . . . . . . . . . . . . . . .

4.5 Results for the SSM to ultrasound group-wise registration. Success Rate (SR) is defined as the ratio of successful results over the total number of registrations. Individual success rate for each vertebra and also the Group Success Rate (GSR) for all vertebrae are reported. A registration is considered a success (GSR) only if the final TRE is less than $3.0 \mathrm{~mm}$ for each vertebra. . . . . . . . . . . . . . .

4.6 T-test probability values for comparing pairwise vs. groupwise registration . . . . . . . . . . . . . . . . . 


\section{List of Figures}

1.1 From left to right: L2-L4. Due to the overlap between the superior and inferior articular processes, as highlighted in the boxes, it is impossible to visualize the superior articular process in US images. . . . . . . . .

1.2 Example of ultrasound images of the lumbar spine in the transverse plane. ........................... 4

1.3 The proposed surgical system for percutaneous spinal needle injections. 7

2.1 A printed model of the human lumbar vertebra. . . . . . . . . . . . . 12

2.2 A surface representation of the L2-L4 vertebrae (top right) with views of the transverse (top left), Coronal (bottom left) and sagittal (bottom right) orientations. . . . . . . . . . . . . . 13

3.1 Outline of the atlas construction method from a set of CT images. . 24

3.2 Outline of statistical atlas to ultrasound registration method. . . . . 25

3.3 Transverse (left), sagittal (center), and coronal (right) slices of the original US volume overlayed with the bone contours of the misaligned (top) and registered (bottom) atlas volumes. . . . . . . . . . . .

3.4 (a) Sagittal, and (b) coronal views of the registered L3 atlas to the 3D ultrasound volume. The five red circles illustrate the landmark positions. 29

4.1 Outline of the SSM construction method from a set of CT images. . . 35

4.2 Registration workflow. . . . . . . . . . . . . . . 37

4.3 Detailed Description of the (a) Rigid and (b) Deformable Registration Blocks for Pairwise Registration . . . . . . . . . . . . . . . . 38

4.4 Detailed Description of the (a) Rigid and (b) Deformable Registration Blocks for Groupwise Registration . . . . . . . . . . . . . . . . 41

4.5 Samples of the segmented CT images acquired from the patients. Top: Axial view; bottom: Sagittal view . . . . . . . . . . . . . . .

4.6 Printed phantom with a realistic spinal curvature. The fiducial markers on the exterior of the box register the acquired ultrasound to the corresponding $\mathrm{CT}$ volume. . . . . . . . . . . . . . . .

4.7 An example of the surface error distribution for SSM to CT registration on the L3 vertebra. . . . . . . . . . . . . . . . . . . . . . . 
4.8 Transverse (left), coronal (center), and sagittal (right) slices of the original US volume overlayed with the bone contours of the misaligned (top) and pairwise registered (bottom) SSM volumes. . . . . . . . . .

4.9 Pairwise registration results for the L2-L4 vertebrae. The five red circles illustrate the landmark positions. . . . . . . . . . . . . .

4.10 Transverse (left), coronal (center), and sagittal (right) slices of the original ultrasound volume overlayed with the bone contours of the misaligned (top) and groupwise registered (bottom) SSM volumes. . .

4.11 The red circles illustrate the position of the surgeon-selected landmarks on the surface of the vertebrae. . . . . . . . . . . . . . . . .

4.12 Graphical representation of the registration results for pairwise and groupwise registration. Phantoms 1, 2 and 3 (left to right) and the L2, L3 and L4 (top to bottom). . . . . . . . . . . . . . . . . .

4.13 Histogram for target registration errors for the pairwise registration. Phantoms 1, 2 and 3 (left to right) and the L2, L3 and L4 (top to bottom). . . . . . . . . . . . . . .

4.14 Histogram for target registration errors for the groupwise registration. Phantoms 1, 2 and 3 (left to right) and the L2, L3 and L4 (top to

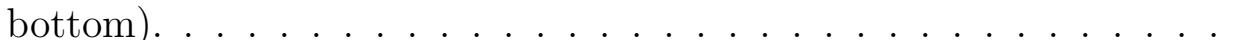

A.1 Parallel implementation of instance generation in GPU. . . . . . . . . 77

A.2 Parallel implementation of ultrasound simulation on the GPU. . . . . 78 


\section{Glossary of Terms}

$\begin{array}{ll}\text { 3D } & \text { 3-dimensional } \\ \text { ASM } & \text { Active Shape Model } \\ \text { CAS } & \text { Computer Assisted Surgery } \\ \text { CMA-ES } & \text { Covariance Matrix Adaptation Evolutionary Strategy } \\ \text { CT } & \text { Computed Tomography } \\ \text { CUDA } & \text { Compute Unifed Device Architecture } \\ \text { GPU } & \text { Graphics Processing Unit } \\ \text { ICP } & \text { Iterative Closest Points } \\ \text { LC2 } & \text { Linear Correlation of Linear Combinations } \\ \text { MI } & \text { Mutual Information } \\ \text { MRI } & \text { Magnetic Resonance Imaging } \\ \text { NCC } & \text { Normalized Cross-correlation }\end{array}$


PCA Principal Component Analysis

RMS Root Mean Square

SNR Signal to Noise Ratio

SR

Success Rate

SSM

Statistical Shape Model

TRE

Target Registration Error

US

Ultrasound 


\section{Statement of Co-authorship}

The work presented in this thesis was done under the supervision of Dr. Purang Abolmaesumin and Dr. Parvin Mousavi, who provided feedback, general direction and corrections to the manuscript. Otherwise the material presented in this document is the original work of the author. Any published (or unpublished) ideas and/or techniques from the work of others are fully acknowledged in accordance with the standard referencing practices. This thesis presents two papers that have already been accepted or in the process within international and top quality journals and conferences. The structure is as follows:

Chapter 3: "Registration of a SSM of the Lumbar Spine to 3D Ultrasound Images", Authors: Siavash Khallaghi, Parvin Mousavi, Ren Hui Gong, Sean Gill, Jonathan Boisvert, Gabor Fichtinger, David Pichora, Dan Borschneck, Purang Abolmaesumi.

Siavash Khallaghi (main author) contributed to the coding, established effective communication between the registration blocks, acquired the ultrasound and designed the experiments.

Sean Gill contributed to the ultrasound simulation code, the design of phantoms and helped with the US acquisition.

Ren Hui Gong contributed to SSM construction code and the CPU implementation of instantiation on the GPU. 
Dr. Jonathan Boisvert provided the segmentation of the vertebrae from patient CT data which was subsequently used as training data for the SSM construction.

Dr. Dan Borschneck selected landmarks on the registration results which was used to measure the target registration error.

Dr. Gabor Fichtinger and Dr. David Pichora provided feedback and helpful discussions during the course of the project.

Dr. Purang Abolmaesumi and Dr. Parvin Mousavi supervised the project and provided feedback, general direction and corrections.

This chapter is to be presented in the upcoming MICCAI 2010 conference.

Chapter 4: "Biomechanically Constrained Groupwise SSM to Ultrasound Registration of the Lumbar Spine",

Authors:Siavash Khallaghi, Parvin Mousavi, Ren Hui Gong, Elvis Chen, Sean Gill, Jonathan Boisvert, David Pichora, Dan Borschneck, Gabor Fichtinger, Purang Abolmaesumi

Siavash Khallaghi (main author) contributed to the coding, established effective communication between the registration blocks, reimplemented the US simulation code on the GPU for a more efficient memory management and to be a more compatible with new versions of CUDA, acquired the ultrasound and designed the experiments. Sean Gill contributed to the ultrasound simulation code, the design of phantoms and helped with the US acquisition.

Ren Hui Gong contributed to SSM construction code and the parallel implementation of instantiation on the GPU.

Dr. Elvis Chen provided the basic parallel implementation of ultrasound simulation on the GPU. 
Dr. Jonathan Boisvert provided the segmentation of the vertebrae from patient CT data which was subsequently used as training data for the SSM construction.

Dr. Dan Borschneck selected landmarks on the registration results which was used to measure the target registration error.

Dr. Gabor Fichtinger and Dr. David Pichora provided feedback and helpful discussions during the course of the project.

Dr. Purang Abolmaesumi and Dr. Parvin Mousavi supervised the project and provided feedback, general direction and corrections.

This chapter is to be submitted to IEEE Transactions on Medical Imaging. 


\section{Chapter 1}

\section{Introduction}

\subsection{Motivation}

Back Pain has been known to be the second inordinately common and disabling condition in the US work force [69]. The majority of the lost productive time is a result of reduced performance while at work and work absence due to pain and discomfort. A recent study, reports the burden of back pain on the US economy to be a high as $\$ 62$ billion [2]. Similar research across the Atlantic, establishes back pain as one of the most costly conditions in the UK economy, estimated as high as $£ 10$ billions a year [50]. Effective forms of treatment include exercises and oral medication [11]. However, [81] reported that nearly $33 \%$ of patients do not respond to oral medication, which results in lack of sleep and limits physical activity. In recent years, improved results for opioid medication have been reported [1], but for some patients oral medication does not provide adequate pain relief. In these cases, an anesthesiologist or a surgeoun performs a spinal needle injection, which targets either the epidural space [78] or the facet joints [51], to deliever a dose of anesthetic agent and/or steroids 
to reduce pain and inflammation. Facet joint injections and epidurals are performed on a regular basis in hospitals and radiological clinics. Historically, these procedures were carried out blind with no image guidance, resulting in a disappointing failure rate of $44 \%$ [47]. However, with the advent of CT and fluoroscopic guidance imaging systems, back pain management has improved tremendousely and has been established as the standard procedure, as $94 \%$ of patients have reported long term pain relief $[56]$.

CT and fluoroscopic images can provide detailed description of the spinal anatomy, the target and the needle location throughout the surgery. However, the use of CT or fluoroscopy requires special equipment and intervention facilities, which are expensive and impose a financial burden to the health care system. Another major drawback to the current standard procedure is that the patient and the surgeon are exposed to ionizing radiation. For a typical spinal injection a minimum of three scans are required. The first scan identifies the target, the second scan shows the needle at the correct position and angle and the third scan is confirms the correct needle insertion. For patients with degraded spines and deformed curvatures, such as scoliotic patients, more images may be needed. Depending on the physician experience and complications, a typical injection may take between 10-45 minutes, with the majority of this time attributed to CT or fluoroscopy imaging. The work presented in this thesis is part of a larger project, which aims to remove the need for intraoperative and preoperative CT and fluoroscopy scans entirely, and to develop an ultrasound-guided minimally invasive spinal intervention system, which would allow the procedure to be performed in a clinic or a physician's office, eliminating the use of expensive specialized facilities and reducing the wait time and travel for patients. In this framework, 
prior knowledge about the spinal anatomy is employed in the form of a statistical shape model (SSM), and bound together with the ultrasound (US) to provide real time guidance for the physician.

\subsubsection{Challenges}

The use of ultrasound as the sole imaging modality during the procedure introduces a set of challenges. To create an ultrasound image, first, the probe transmits an acoustic signal through the anatomy. As this signal propagates through different tissue types, due to impedance mismatch, a portion of the ultrasound wave is reflected back to the ultrasound probe. In the case of the spine anatomy, the density of the bone is significantly larger than the surrounding soft tissue. This often causes full reflection in the US-signal and prevents the US waves from traveling beyond the bone surface.

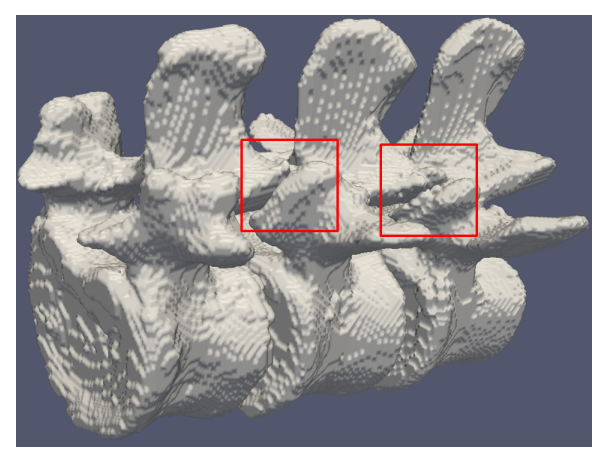

Figure 1.1: From left to right: L2-L4. Due to the overlap between the superior and inferior articular processes, as highlighted in the boxes, it is impossible to visualize the superior articular process in US images.

This phenomenon occludes any anatomy that is beneath the bone surface, making it impossible to visualize the surface of the superior articular process in ultrasound images, as shown in Figure 1.1. Due to the significant reflection occurring at these 


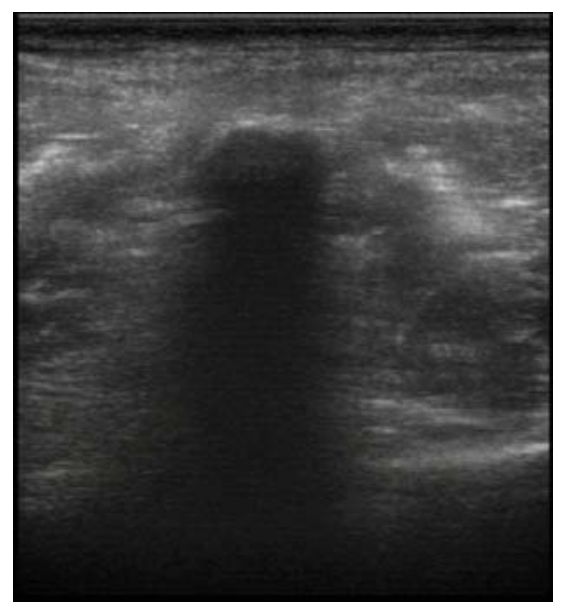

Figure 1.2: Example of ultrasound images of the lumbar spine in the transverse plane.

interfaces, the surface of the bony structure appears bright in the image, but is often blurred and difficult to identify exactly. Additionally, not all bone surfaces are visible in US. If a US signal reflects at a bony and soft-tissue interface that is angled away from the US transducer, the signal will not return to the transducer and the surface will not appear in the US image. Finally, US images contain a significant amount of speckle in the soft-tissue portion of the image. For these reasons, US as a sole modality for guidance is not ideal, as a clear depiction of the location of the needle relative to the target anatomy is essential for a clinically viable guidance system. An example of the ultrasound images of the lumbar spine is shown in Figure 1.2.

A statistical shape model is a mathematical entity, which captures the statistical information, including the mean and variations, of a group of objects. It not only represents the objects within the population of the constructed SSM, but can also produce interpolated objects which did not exist during the training phase. The process of creating new objects is called instantiation. In this project, CT images 
acquired from patients have been used to construct a statistical shape model of the lumbar spine.

The use of CT images as training data for the SSM representation of the spinal column adds an additional challenge to the registration problem. In order to increase patient's comfort during CT imaging, the patient is asked to lay in the supine position. On the other hand, the procedure is performed with the patient laying in the prone or sitting position. This introduces a change in the curvature of the spine, which can only be accounted for if a separate model for each vertebra is constructed and treated independently from the neighboring vertebrae. Furthermore, constructing a statistical shape model that can be an adequate representation of a population of the vertebrae is by no means trivial. Extreme variations in shape, sharp edges inherent to the anatomy and the presence of outliers make this a challenging task.

In this thesis, a novel biomechanically constrained registration of statistical shape models to 3D ultrasound images of the spine is presented. To the best of our knowledge, this is the first report of a groupwise registration of a statistical shape model to 3D ultrasound without prior segmentation or preprocessing in the ultrasound data. The registration technique is validated on data from patient-based phantoms. These phantoms have been designed to preserve a realistic representation of the curvature of the spine.

In order for the registration to be usable in a real clinical setting, the registration must be performed in real-time. This time-constraint imposes an additional challenge, since most deformable and multi-modality registrations tend to be both time-consuming and computationally expensive. Since most intervention facilities lack high performance computing hardware, parallel implementation on the Graphics 
Processing Unit (GPU) has been proposed as an affordable alternative for real-time applications [67].

\subsection{Proposed Image Guided Surgical System}

Figure 1.3 illustrates the proposed system for facet joint injections. The patient is asked to lay in the prone position. An ultrasound volume is acquired using a tracked linear probe.

In order to represent the anatomy of the spine, a statistical shape model is created prior to the procedure. This prior knowledge is passed to the guidance system, and is fused with the patient's ultrasound using an image registration block. With the statistical shape model registered to the patient ultrasound, the position and orientation of the ultrasound data and a tracked needle is known with respect to the anatomy. The registration block is the main focus of this thesis, which is explained in detail in Chapters 3 and 4 .

The registration technique, takes advantage of two previous projects developed within our group, and have been presented by Gong et al. [26] and Gill et al. [22]. Gong et al. [26] developed a statistical shape model based on B-spline deformation fields and validated it on the distal radius CT images. The work presented in this thesis also further extends the work presented by Gill et al. [22], which develops a method for the groupwise registration of US and CT images of the spine. 


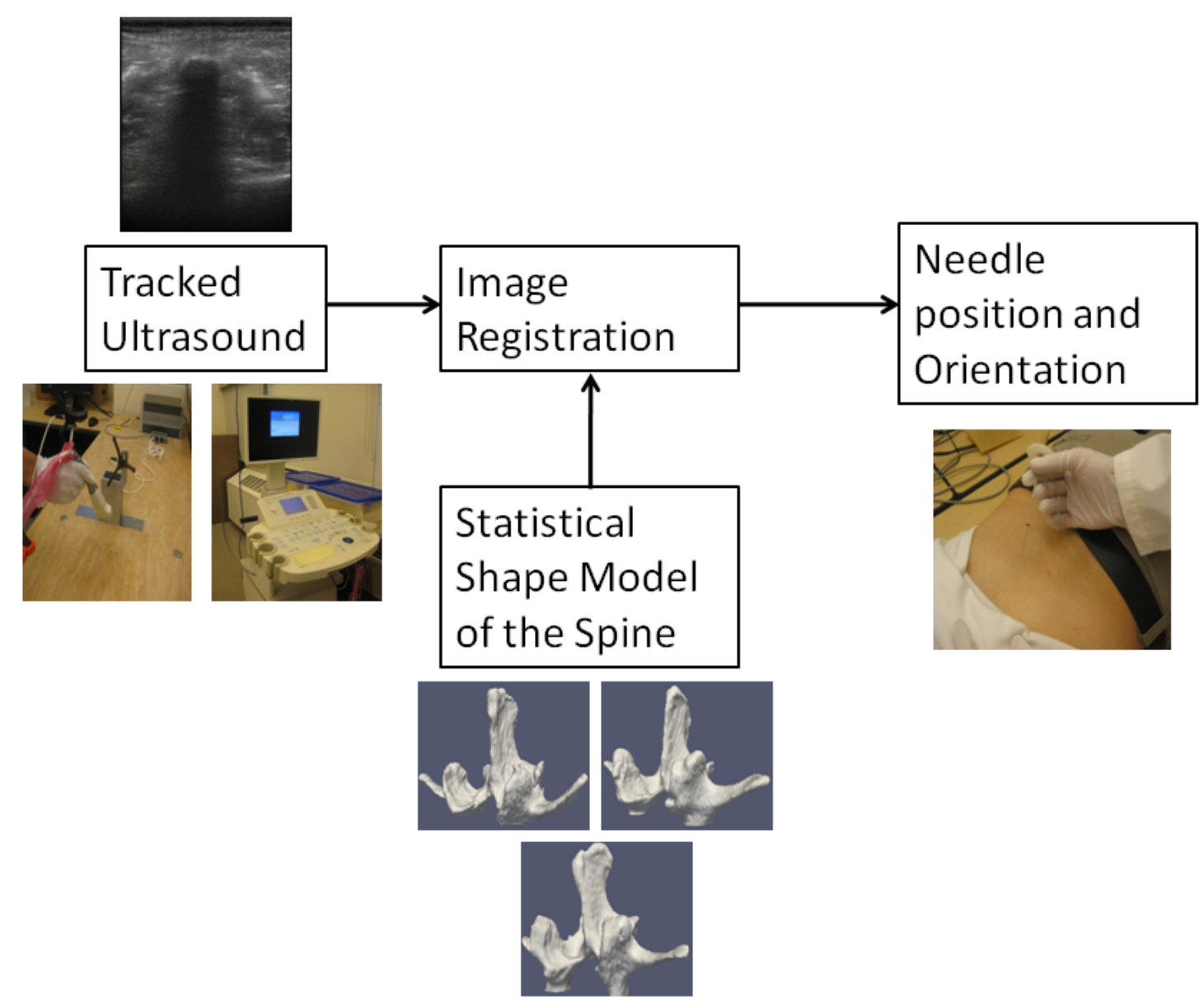

Figure 1.3: The proposed surgical system for percutaneous spinal needle injections.

\subsection{Thesis Objectives}

The work presented in this manuscript is part of a larger project, which aims to develop an ultrasound-based guidance system for percutaneous spinal interventions. The goal of this work is to develop a statistical shape model to ultrasound registration technique, which is both robust and accurate in terms of finding the correct shape, position and orientation of the spine column during the procedure. This registration 
method, when used in conjunction with the US image based guidance system, would reduce the need for intra-operative or pre-operative $\mathrm{CT}$ and fluoroscopy in spinal needle injections. With no or little X-ray-based guidance being necessary, the exposure of both the patient and physician to ionizing radiation would be substantially reduced. Furthermore, this will eliminate the need for special interventional facilities, which would decrease the cost of such interventions dramatically. This objective can be accomplished through the following:

- To construct a statistical shape model of the lumbar vertebrae (L2-L4), which can effectively span the anatomical shape variations in a population and can be an adequate representation of the lumbar vertebrae.

- To develop a groupwise SSM to ultrasound registration framework, which can retrieve the shape, position and orientation of each vertebra.

- Validation to determine the accuracy and robustness of the proposed method.

\subsection{Contributions}

In this thesis, I have:

- constructed the first volumetric SSM of the lumbar vertebra, which has both outline (geometry) and intensity information.

- developed a framework for the groupwise registration of statistical shape models of the lumbar spine to 3D ultrasound images, which can retrieve the shape, position and orientation of the L2-L4 vertebrae. The algorithm does not require the segmentation of the bone surface in the ultrasound data. Furthermore, since 
the distance between neighboring vertebrae varies between different patients, a modified biomechanical model is presented that constrains the relative motion of the vertebra models to anatomically realistic orientations and positions through the use of a biomechanical model.

- implemented the registration algorithm on the GPU to significantly reduce the computation time.

- validated the proposed registration framework on data collected from phantoms designed to preserve the shape and curvature of the human spine.

\subsection{Organization of Thesis}

This thesis, presented in the manuscript format, is based on two papers which constitute Chapters 3 and 4 of this document. Chapter 3 , is to be presented in the Medical Image Computing and Computer Assisted Intervention (MICCAI 2010) conference [37], and Chapter 4 is for submission to IEEE Transactions on Medical Imaging. The thesis is divided into five chapters, which discuss the SSM construction method, the registration technique and the validation procedure. The organization is as follows:

Chapter 2 provides a brief overview on previous work done on statistical shape models and images registration.

Chapter 3 is a feasibility study on the registration of a statistical shape model of a single vertebra to 3D ultrasound images. 
Chapter 4 further extends the previous chapter to a groupwise registration of the statistical shape model of the L2-L4 vertebrae to ultrasound images. This chapter provides a detailed description of the construction of the statistical shape model, the parallel implementation on the graphics processing unit and the biomechanical model. Quantitative results are given for patient-based phantoms. The implications of the results are discussed throughout the chapter.

Chapter 5 presents the key conclusions of the thesis and possible areas of future work that can aid in preparing this work for clinical use.

Appendix A is a brief description of the parallel implementation of SSM instantiation and ultrasound simulation on the GPU. 


\section{Chapter 2}

\section{Background}

In this chapter the important aspects of the anatomy of the spine for the targeted clinical application are examined, and the prior work on SSMs and multi modal image registration are presented.

\subsection{Anatomy of the Lumbar Vertebrae}

Depending on the objective of the procedure, percutaneous spinal needle injections have specific target locations for pain management. The main focus of this work is on facet joint injections. The facet joint is located at the overlap of the inferior and superior articular processes on neighboring vertebrae [16]. The most common injection sites are at the overlap of the L2-L3 and L3-L4 vertebrae. An image of the lumbar vertebra is shown in Figure 2.1. For a successful facet joint injection, the anesthetic dose must be released close to the target region. Complications may arise if the needle tip punctures too deep into the tissue causing nerve damage and increased pain [16]. 


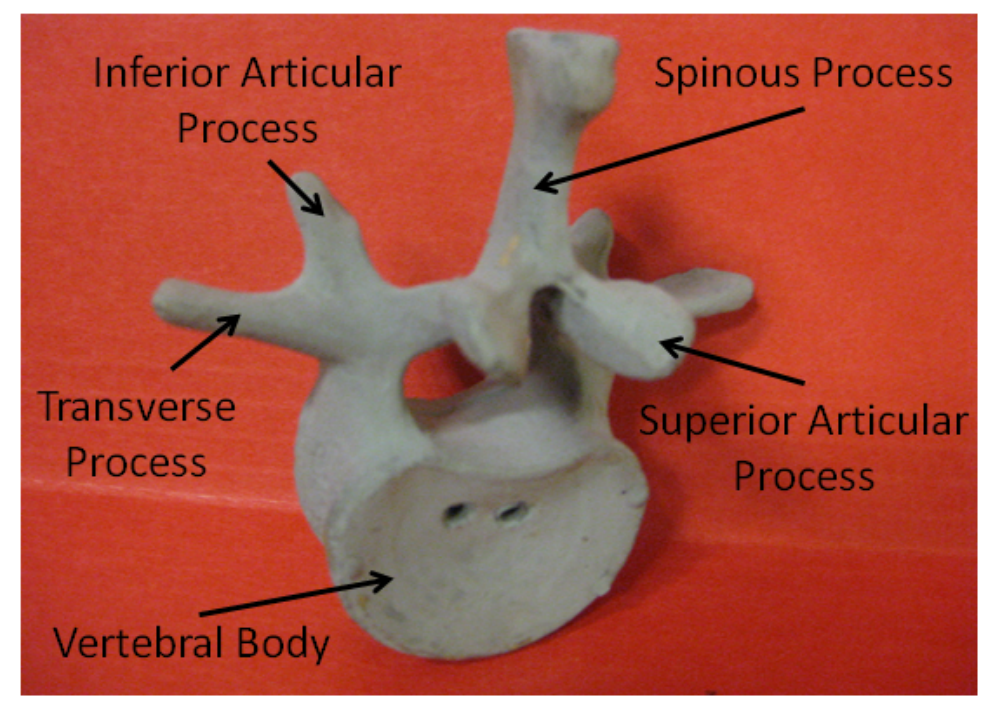

Figure 2.1: A printed model of the human lumbar vertebra.

While the articular processes are the main target of facet joint injections, the spinous process is an important landmark in spinal needle injections. From a physician's perspective, due to its proximity to the skin surface, the spinous process gives a rough estimate on the location of the vertebra. A surface model of the L2-L4 lumbar vertebrae is shown in Figure 2.2.

\subsection{Statistical Shape Models}

SSMs are a very powerful modeling tool for anatomical structures that provide a compact representation of statistical variation in shape across a population of training samples. Recently, SSMs have grown in popularity in a wide range of image processing applications: segmentation [29, 82], statistical analysis [6] and registration [43, 72]. As mentioned in section 1.2, prior knowledge of the anatomy is introduced through SSMs, 

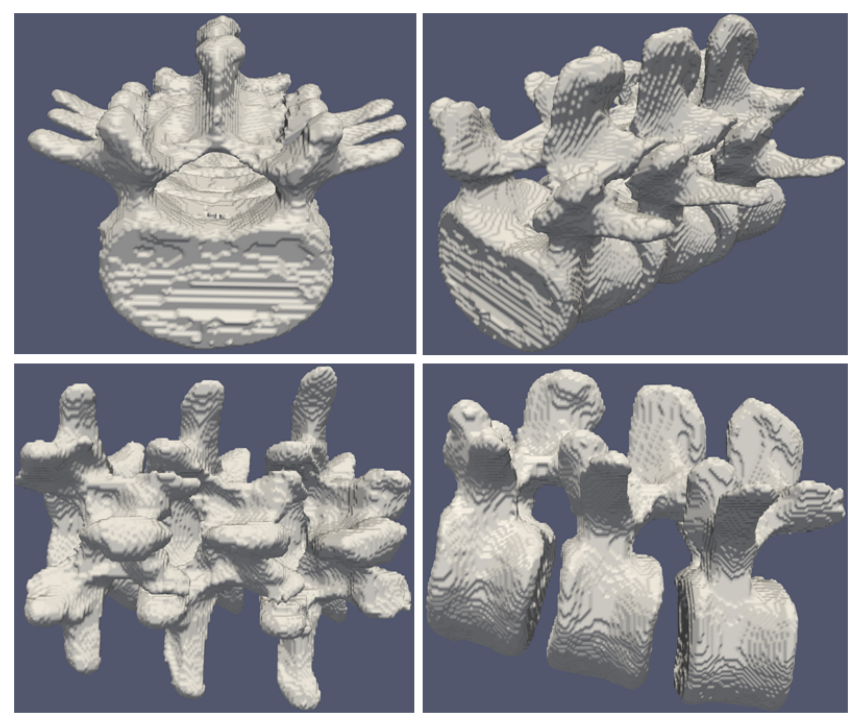

Figure 2.2: A surface representation of the L2-L4 vertebrae (top right) with views of the transverse (top left), Coronal (bottom left) and sagittal (bottom right) orientations.

which removes the need for ionizing CT scans. This is in line with a number of papers which also propose SSMs as an alternative to preoperative CT imaging [21, 4, 58]. Existing SSMs can be divided into two categories: geometrical SSMs $[4,32,53,66]$, which describe the outline of images, and volumetric SSMs [26, 35], which model both the internal intensity and geometrical information.

Because geometrical SSMs only represent the boundary of the population, they are computationally less expensive in comparison to volumetric SSMs. Talib et al. [71] have investigated the feasibility of ultrasound registration to geometrical SSMs of the femur. Barratt et al. [4] and Foroughi et al. [21] have built a SSM for the femur and pelvis, which is subsequently repositioned and deformed to fit a cloud of bone surface points extracted from a set of tracked US images. Similarly, Rajamani et al. [64] constructs a SSM of the femur from sparse point-sets and shows that this 
point-set representation can adequately describe the anatomy. Bookstein et al. [8] use landmarks to capture the important geometric features. The active shape model (ASM) of Cootes et al. [14] represents an object's geometry as a dense collection of boundary points. Also Cootes et al. [15] have augmented their statistical models to include the variability of the image information as well as shape. Kelemen et al. [36] have used a spherical harmonic (SPHARM) decomposition of the object geometry. Recent publications have also explored methods towards constructing a statistical shape model using nonrigid deformation of a template mesh [31].

While geometrical SSMs are computationally less expensive, they are prone to bone surface segmentation errors in ultrasound data. This has shifted the research to volumetric SSMs. Thirion's demons algorithm, which solves local Partial Differential Equations (PDEs) to compute the deformation field and builds a SSM of the femur. The feasibility of ultrasound registration to volumetric SSMs has been previously investigated [37]. Particularly in the spinal column, a number of papers strive to model various anatomical structures. Gong et al. [26] builds a B-spline SSM of the femur and shows it to be an adequate representation of the distal radius. Jurcak et al. [35] have proposed a B-spline SSM of the quadratus lumbarom muscle and have used it for segmentation. All of the proposed volumetric SSMs so far describe anatomical structures that have a fairly smooth surface. However, in the case of human vertebrae, due to extreme variations in shape and presence of sharp corners, building a statistical shape model for the vertebrae is shown to be a challenging task and is still an active field of research [48]. Due to the complexity of the demons algorithm and solving local PDEs, I have used the B-spline transform as the basis for the SSM of the lumbar vertebrae. 


\subsection{Multi Modal Image Registration}

Image Registration is a key block in integrating the preoperatvie CT models with intraoperative ultrasound. Previously in the literature, multi-modality registration has been used for a wide variety of diagnostic [32] and therapeutic purposes, including: orthopedic procedures [5, 54], neurosurgical interventions [24], abdominal procedures [45] and cardiovascular operations [34, 38]. However, accurate registration of ultrasound and CT images has proven to be a difficult problem [34, 38], due of the low Signal To Noise Ratio (SNR) of ultrasound images, the presence of US imaging artifacts and the fact that the same anatomy can presents itself as quite different structures in CT compared to ultrasound. For this reason, most of the existing algorithms preprocess either of the modalities to reduce the artifacts and to increase the similarities in either images. For example, Roche et al. [65] have registered 3D US to MRI by using both intensity and gradient information. Similarly, Leroy et al. [45] have registered $\mathrm{CT}$ with freehand 3D US, by preprocessing the CT images to boost edges and the US images to remove the speckle and shadows. Pre-operative image gradient/edge information has also been used by Pluim et al. [61]. Huang et al. [34] have preprocessed both modalities to preserve only features which appear similar in either images. Penney et al. [60] have remapped both MRI and freehand 3D US to a vessel probabilistic representation before co-registering.

The existing registration techniques can be divided to feature-based and intensitybased registration. Feature-based methods strive to solve the registration problem by establishing a correspondence between specific anatomical structures, i.e features. However, in most applications, finding a set of features which is both visible in all images and can be robustly segmented is a challenging task. Porter et al. [62] have 
presented a method for registration of US and MR images of the vasculature in which major vessels in the volumes are treated as anatomical landmarks. The vessels have been extracted by thresholding the images and applying morphological kernels. Firle et al. [20] have proposed a registration of preoperative US and CT images of the prostate using manual segmentation of the urethral surface and subsequent registration using Iterative Closest Points (ICP). In a similar method, US and MR images of the liver have been registered together using ICP optimization method on the centerline of vessels [42].

On the other hand, intensity-based registrations for soft-tissue are popular as they do not require the segmentation of surface organs. Especially, in ultrasound images, this segmentation can be both time-consuming and cumbersome, as the images are usually blurred and poorly defined. Shao et al. [68] have proposed to extract the bone surface of the pubic arch from MR image and register it to the US volume. Huang et al. [33] have presented a similar method, designed to register US and CT images of the rib cage as a form of initialization for US to MR registration of the heart. In another approach, proposed by Brendel et al. $[9,10]$ and Winter et al. [79], it is assumed that the brightest points in US images are usually caused by reflection of the signal on the bone surface. After extracting the bone surface from the CT volume, the surface is degraded to only what is visible in the US data, followed by the registration. A recent approach for intensity-based registration focuses on ultrasound simulation from CT data. Wein et al. [77] has been the first to report this approach for rigid US to CT registration of the head and neck. They have further extended this work to affine registration of soft tissue [76], which is used in the current thesis. Our research group has further extended the simulation-based registration method [22], in which 
a biomechanically constrained groupwise registration of the lumbar vertebrae to 3D ultrasound images is performed.

\subsection{GPU-based Acceleration}

A significant improvement in the GPU technology, is the advent of the Compute Unified Device Architecture (CUDA). CUDA is the computing engine in nVidia GPUs that is accessible to software developers through industry standard programming languages such as $\mathrm{C}$, thus allowing developers to build applications without requiring in-depth graphics programming knowledge. In this model, the GPU is viewed as a coprocessor to the host $\mathrm{CPU}$, while maintaining its own device RAM, shared between the host CPU through global memory. CUDA supports scalable programming, in which computation is performed on a set of blocks (grouped together to form a grid) that run in parallel. Each block contains a number of threads, the basic computation unit. The GPU, performs data-parallel portions of the application by means of kernel on collaborative threads. Running the proposed registration algorithm on a desktop personal computer, will take days, rendering it useless for real-time clinical applications. To speed up the registration, we have implemented a parallel version of our algorithm on an nVidia graphics card and have demonstrated significant reduction in the computation time.

Run-time is often the limiting factor in most Computer Assisted Surgery (CAS) systems. Since the execution-time in most intra-operative applications must be in real-time, a number of papers have investigated the parallel implementation of the graphics processing unit for both segmentation and registration $[28,57,67,80]$. Narayanaswamy et al. [59] segment the vessel laminae in a parallel implementation of 
a robust hypothesis test followed by an adaptive region growing using a ray casting method. Ozcelik et al. [57] implement a parallel version of the Mutual Information (MI) metric to perform SSM-based segmentation of the MR-brain images. Lefohn et al. [44] perform level set segmentation by solving local partial differential equations on the GPU. This is done by only updating voxels that are on or near the iso-surface. All of the mentioned papers report a drastic computation gain by using the parallel implementation on the GPU.

Typically the most time-consuming portions of the registration are the transformation of the moving image and the calculation of the similarity metric. Depending on the application, either of these blocks may be the bottleneck for the registration algorithm. Crookes et al. [17] implements an affine registration on the graphics processing unit. Ozcelik et al. [57] present a parallel implementation of the demons algorithm. Rehman et al. [73] is another example of GPU-based deformable registration. In this paper, a multi-resolution approach is implemented to solve the Optimal Mass Transport for non-rigid registration and is implemented on the GPU for a faster convergence. The above mentioned papers, also show a significant decrease in the registration time using GPUs.

Multi-modality registration between $\mathrm{CT}$ and US images, due to to the different nature of input images, tends to be a time-consuming and complex task. Where feasible, papers have investigated GPUs as an affordable solution to the registration problem. Kutter et al. [40] presents a modular design for ray-casting ultrasound simulation from CT images with various degrees of complexity on the GPU, which is the core of many multi-modality registration algorithms. In another paper, Kutter et al. [41] performs a multi-modality registration between ultrasound and CT images on 
the GPU using a variation of the Linear Correlation of Linear Combinations (LC2) metric, originally proposed by Wein et al. [76], by bringing the two volumes into initial alignment using a global Normalized Cross Correlation Value (NCC) and then exchanging local NCC values for final refinement. While these papers provide a very complex and accurate simulation of ultrasound, for this project, due to lack of soft tissue information in the lumbar SSMs, a simplified implementation of US-simulation on the GPU is presented. 


\section{Chapter 3}

\section{Registration of a SSM of the \\ Lumbar Spine to 3D Ultrasound}

\section{Images}

\subsection{Overview}

Motivation: Spinal needle injections are technically demanding procedures. The use of ultrasound image guidance without prior $\mathrm{CT}$ and $\mathrm{MR}$ imagery promises to improve the efficacy and safety of these procedures in an affordable manner. Methodology: We propose to create a statistical shape model of the lumbar spine and warp this atlas to patient-specific ultrasound images during the needle placement procedure. From CT image volumes of 35 patients, statistical shape model of the L3 vertebra is built, including mean shape and main modes of variation. This shape model is registered to the ultrasound data by simultaneously optimizing the parameters of the model

To be presented in MICCAI 2010, Beijing, China. 
and its relative pose. Ground-truth data was established by printing 3D anatomical models of 3 patients using a rapid prototyping. CT and ultrasound data of these models were registered using fiducial markers. Results: Pairwise registration of the statistical shape model and 3D ultrasound images which, according to our clinical collaborators, led to a mean target registration error of $3.4 \mathrm{~mm}$, while $81 \%$ of all cases yielded clinically acceptable accuracy below the $3.5 \mathrm{~mm}$ threshold.

\subsection{Introduction}

Spinal needle injection is widely applied in analgetic and diagnostic purposes [3]. In the management of back pain, epidural anesthesia, facet joint injections and nerve blocks are common forms of these interventions, performed in great numbers in hospitals and radiology clinics. Back pain is the second most common reason for a visit to the physician. In the United States alone, approximately $90 \%$ of adults will experience back pain at some point in their life. Nearly $50 \%$ of the current working population has reported some history of back pain; this is the leading cause for missed work time and seriously degrades on-the-job performance [2]. In back pain management, accessing the lumbar epidural space presents major difficulties. The success rate of proper needle insertion is a dismal $60 \%$ after 10 attempts [75] when no guidance is used, clearly an inadequate practice. Contemporary radiological needle guidance with CT and fluoroscopy requires specialized facilities often unavailable to patients living in rural areas, and involve X-ray radiation. In search of a more accessible, portable, and non-toxic imaging alternative, ultrasound guidance has been considered. Watson et al. [74] and Klocke et al. [39] targeted the L3-L4 and L2-L3 interspace, a challenging procedure that, if performed inaccurately, damages the spinal cord. The results 
suggested that ultrasound as a solo guidance is inadequate. To address this issue, combination of ultrasound with CT has been proposed [55, 79, 22]. In these studies, the auxiliary information is necessary for guidance, but as stated above, either unobtainable or involves ionizing radiation. Hence, the use of statistical shape models is a logical alternative.

Previously in the literature, statistical shape models (or atlases) have been considered as an alternative to pre-operative CT scans $[72,32,53]$. These atlases are generally divided into two main categories: those that describe the outline of objects (geometrical atlases) $[72,32,53,21,66]$ and those that contain both the geometrical and internal density distribution of the object (volumetric atlases) [4, 26, 35]. As an example of geometrical atlases, [6] captures the variations in pose of each vertebra and builds a model for the curvature of the spine. The feasibility of ultrasound registration to a statistical atlas of femur has been previously investigated [71]. In [21] a geometrical atlas to ultrasound registration of the pelvis is performed. While geometrical atlases are computationally less expensive, they are prone to bone surface segmentation errors in ultrasound data.

Our contribution is the first report of a volumetric vertebral atlas and its registration to 3D ultrasound without prior segmentation. We demonstrate a feasibility study on the L3 vertebrae, one of the most problematic anatomical sites in spinal pain management. In departure from the prior art using tetrahedral mesh and Bernstein polynomials [66], we employ a simple but generic approach based on the B-spline deformable transformation that allows for straightforward extension for an ensemble of vertebrae and relevant structures $[4,26,35]$. 


\subsection{Method}

\subsubsection{Construction of the Statistical Shape Model}

Figure 4.1 demonstrates the atlas model construction process. A set of CT images, acquired from 38 patients (19 male and 19 female), was used in this study. Data was collected under the approval of the research ethics board, and the patients involved provided informed consent for this data to be used in the research. Using ITKSnap, the L3 vertebra was semi-automatically segmented from the CT volumes and resampled to a a $120 \times 200 \times 100$ volume with an isotropic spacing of $0.6 \mathrm{~mm}$. The patient data was divided into two groups: 35 for constructing the atlas (hereafter referred to as training data), and 3 for validation (two male and one female). One of the CT volumes for atlas construction was chosen as the template $I_{\mathrm{t}}$. Each training example, $I_{k}$, is registered to the template by a rigid registration followed by a Bspline deformable registration, such that $I_{\mathrm{t}} \approx T_{\text {def }}^{k}\left(T_{\text {rigid }}^{k}\left(I_{k}\right)\right)$, where $T($.$) denotes$ a transform. B-spline registration is performed in a $40 \times 30 \times 30$ grid using Mattes Mutual Information metric. To reduce the deformable registration time, a three stage multi-resolution approach is implemented. Each deformable registration took between two and four hours on a Core 2 Quad CPU machine with $2.4 \mathrm{GHz}$ speed and $3 \mathrm{~GB}$ of RAM. With the deformable transform of all the training examples known with respect to the template, principal component analysis (PCA) is performed to construct the statistical atlas for the L3 vertebrae.

After the atlas is constructed it can be used to generate new instances of the population. A new instance of the atlas, defined by the deformation vector, $D_{n e w}$ ,can be produced by a linear combination of the mean deformation vector, $\bar{\phi}$, atlas 


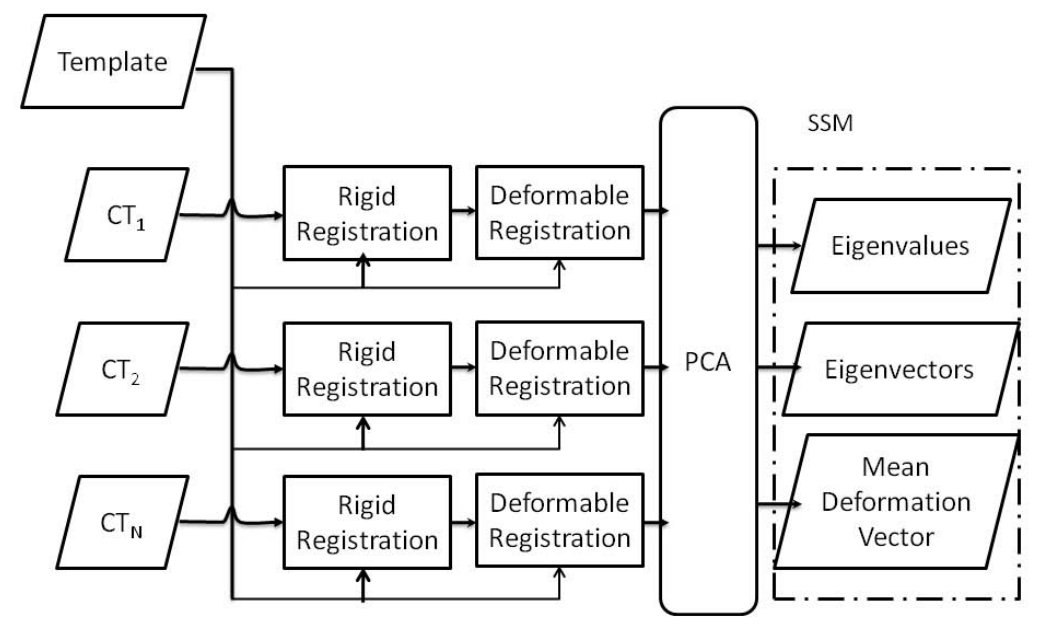

Figure 3.1: Outline of the atlas construction method from a set of CT images.

weights, $w_{i}$, and the eigenvectors of the covariance matrix generated from all the deformation fields, $v_{i}$, as follows:

$$
D_{\text {new }}=\bar{\phi}+\sum_{i=1}^{N} w_{i} v_{i}
$$

\subsubsection{Statistical Atlas to Ultrasound Registration}

The registration framework is shown in Figure 3.2. First, the mean shape of the L3 atlas is rigidly registered to the 3D ultrasound volume by simulating ultrasound images from the atlas, and performing an intra-modality registration with the 3D ultrasound images [76]. The similarity metric used is the Linear Correlation of Linear Combinations $\left(\mathrm{LC}^{2}\right)[76]$ between the actual and the simulated ultrasound images, and is computed from the atlas volume using:

$$
L C^{2}=\frac{\sum(U(x, y)-f(x, y))^{2}}{M \times \operatorname{Var}(U)}
$$




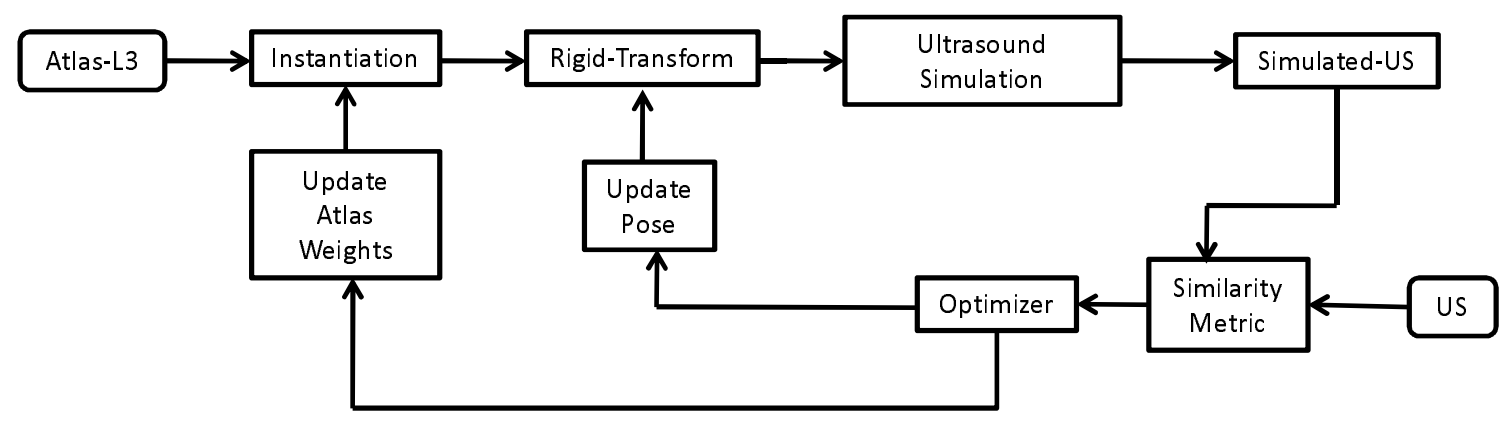

Figure 3.2: Outline of statistical atlas to ultrasound registration method.

where $f$ is the simulated ultrasound image, $U$ is the actual ultrasound image and $M$ is the number of overlapping voxels between the ultrasound and the atlas volume. We used the Covariance Matrix Adaptation Evolution Strategy (CMA-ES) as the optimization method [30]. Following the initial rigid registration, we performed deformable registration of the atlas model to the 3D ultrasound data by simultaneously creating new instances of the atlas and updating the rigid transformation and the atlas parameters, while optimizing the $\mathrm{LC}^{2}$ similarity metric. 


\subsection{Experiments and Results}

\subsubsection{Statistical Shape Model}

We investigated whether the statistical shape model generated from L3 can span the space of shape variations in the patient population. For this purpose, we used a leave-one-out cross registration with the CT data. In each step, we left out one of the patient CTs for testing, created the atlas from the rest of the data, and registered the created atlas to the remaining patient CT. This process was repeated by selecting each CT data once as the testing data. Throughout the process, the template was held constant. To validate the model, we used the first 12 eigenvectors which covered $95 \%$ of the total modes of variation. The registration process used Mattes Mutual Information and deformable B-Spline registration, in conjunction with the CMA-ES optimizer to solve for the atlas model and rigid registration parameters.

As in [72], the Root Mean Square (RMS) error between the closest surface points of registered atlas and the test data was used to measure the capability of the atlas to capture the deformations within the patient population. The mean error across all registrations was $0.89 \mathrm{~mm}$ with a standard deviation of $0.19 \mathrm{~mm}$.

\subsubsection{Registration of the Atlas to Ultrasound Images}

In a second set of experiments, we aim to show how well the constructed atlas can be deformed and reoriented to match the shape and position of the patient vertebra using the acquired 3D ultrasound images. In each registration, we simultaneously optimized 12 shape and 6 rigid parameters, which correspond to the first 12 PCA eigenvectors and the pose respectively. 
The three excluded CT volumes from the atlas generation process (see Section 3.3.1) were used to construct 3D CAD models of the entire lumbar spine, including L1 to L5. These models were printed using a Cimetrix 3D shape printer (Cimetrix Solutions, Oshawa, ON, Canada). Three spine phantoms were constructed by submerging these models in an agargelatine-based tissue phantom which was designed to simulate the appearance of soft tissue in ultrasound. A high-resolution CT image $(0.46 \times 0.46 \times 0.625 \mathrm{~mm})$ and an ultrasound volume were acquired from each phantom. The ultrasound volume was reconstructed from a freehand sweep with an L14-5/38 linear-array transducer (Ultrasonix, Richmond, BC, Canada) operating at $6.6 \mathrm{MHz}$ with an imaging depth of $5.5 \mathrm{~cm}$. The probe was tracked using an Optotrack Certus System (Northern Digital Inc., Waterloo, ON, Canada) and calibrated using an N-wire phantom [13]. The phantom CT and ultrasound volumes were aligned using fiducial markers mounted on the exterior of the phantom box. The position of these fiducials in the ultrasound coordinate system was identified using a calibrated stylus pointer.

The atlas mean shape and the ultrasound volumes were brought to an initial position by rigidly registering the mean shape to the corresponding phantom $\mathrm{CT}$ volume. For each phantom, thirty experiments were performed with perturbing the mean shape using a transformation generated from a uniform random distribution in the interval of $[0,10] \mathrm{mm}$ translation along each axis and $[0-10]^{\circ}$ rotation about each axis. The registration parameters were then optimized as it was discussed in Section 3.3.2.

To evaluate the accuracy of the registration, an expert orthopedic surgeon and an anesthesiologist were asked to identify five corresponding landmarks, three on the 


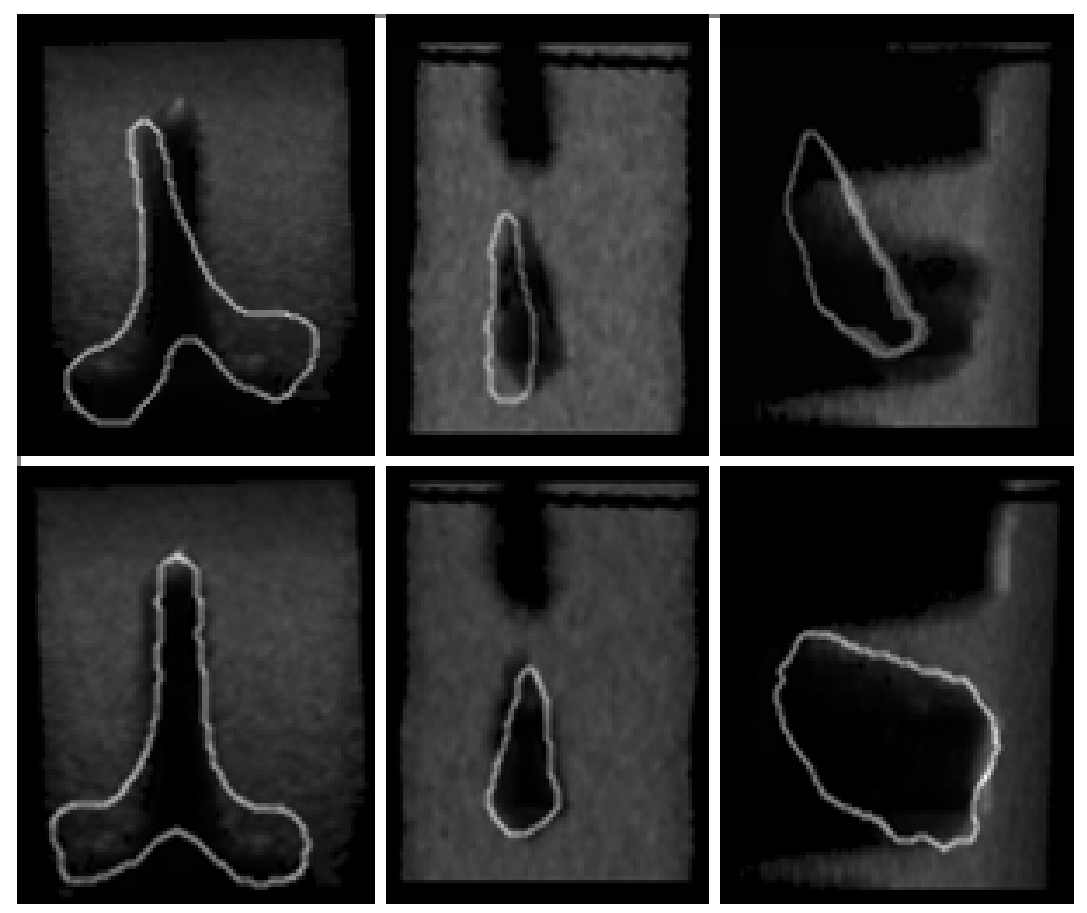

Figure 3.3: Transverse (left), sagittal (center), and coronal (right) slices of the original US volume overlayed with the bone contours of the misaligned (top) and registered (bottom) atlas volumes.

spinous process and two on the target facet joints shown in Figure 4(b), on the registered atlas, the ultrasound volume and the corresponding CT. The average distance of these five landmarks was chosen as a measure of the final Target Registration Error (TRE). A registration was considered failed if the final TRE is more than $3.5 \mathrm{~mm}$, as the clinically accepted error. Registration results are shown in Table 3.1, with the mean and standard deviation calculated over all the thirty experiments. Also an example of the initial misalignment and the registration result is depicted in Figure 3. Figure 4 shows an overlay of the registered atlas with the ultrasound volume. 


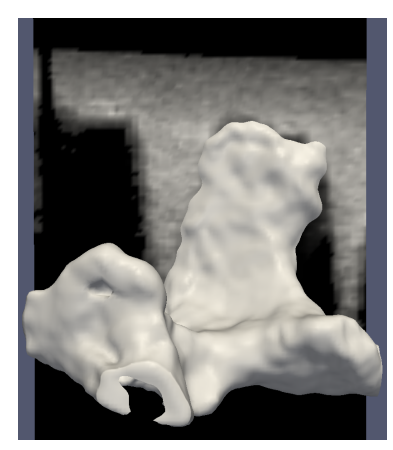

(a)

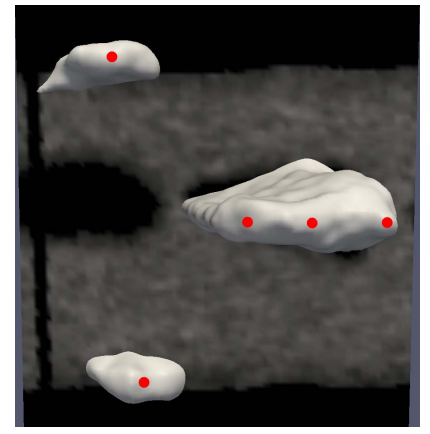

(b)

Figure 3.4: (a) Sagittal, and (b) coronal views of the registered L3 atlas to the 3D ultrasound volume. The five red circles illustrate the landmark positions.

\begin{tabular}{cccc}
\hline Phantom & $\begin{array}{c}\text { Mean Landmark Error } \\
\mathrm{mm}\end{array}$ & $\begin{array}{c}\text { Std } \\
\mathrm{mm}\end{array}$ & $\begin{array}{c}\text { SR } \\
\%\end{array}$ \\
\hline 1 & 3.38 & 0.42 & $81 \%$ \\
2 & 3.48 & 0.33 & $79 \%$ \\
3 & 3.25 & 0.45 & $82 \%$
\end{tabular}

Table 3.1: Registration results for the atlas to US registration. SR (Success Rate) is defined as the ratio of the registrations where the overall TRE is less than $3.5 \mathrm{~mm}$. SR is presented for each phantom with the maximum initial misalignment of $10 \mathrm{~mm}$.

As seen in Table 3.1, the average TRE is less than $3.5 \mathrm{~mm}$ with an average success rate of $81 \%$ for all three phantoms. Our preliminary results for the registration of atlases created from L2 and L4, the neighboring vertebrae to L3, to 3D ultrasound volumes, with the same patient data set, have shown TRE below $3.2 \mathrm{~mm}$ based on five landmarks per vertebrae. The registration results satisfy clinical requirements for facet joint injection. They also demonstrate the feasibility of using volumetric atlases for the registration of a patient spine to 3D ultrasound data. This can enable the use of ultrasound image guidance for spinal interventions without prior $\mathrm{CT}$, to improve 
the efficacy of these procedures in an affordable manner.

On going research is aimed at addressing several goals: i) Run time; currently, a single registration of the atlas model to ultrasound data, implemented in unoptimized $\mathrm{C}++$ code, takes in the order of hours on a $2.3 \mathrm{GHz}, 16$-core Pentium machine with 16 GB of RAM. Future efforts will focus on speeding up the computation, specifically by implementing the algorithm on graphics processing units (GPUs). Recently released open-source software for GPU implementation of B-spline interpolation and registration will facilitate achieving this goal; ii) Atlas generation bias; the current atlas generation process is potentially biased towards the chosen template. An alternative would be to use groupwise atlas generation methods that have become widely popular recently, especially in neuroimaging research; iii) Spine registration; at the moment, the method only registers the atlas of a single vertebra to 3D ultrasound volumes. Registration of an atlas of the entire (or partial) spine with the ultrasound data would provide better contextual information to the physician for intervention. 


\section{Chapter 4}

\section{Biomechanically Constrained}

\section{Groupwise SSM to Ultrasound}

\section{Registration of the Lumbar Spine}

\subsection{Overview}

Spinal needle injections for back pain management are frequently carried out in hospitals and radiological clinics. Currently, these procedures are performed under fluoroscopy or CT guidance in specialized interventional radiology facilities. As an alternative, the use of inexpensive ultrasound image guidance promises to improve the efficacy and safety of these procedures. We propose to eliminate or reduce the need for ionizing radiation, by creating a statistical shape model of the lumbar vertebrae, and registering it to 3D ultrasound volumes of patient using a groupwise registration algorithm. From a total of 35 patient CT volumes, a statistical shape model of the

To be submitted to IEEE Transactions on Medical Imaging 
L2, L3 and L4 vertebrae is built, including the mean shape, and principal modes of variation. The statistical shape model is registered to the 3D ultrasound by interchangeably optimizing the model parameters and their relative poses. We also use a biomechanical model to constrain the relative motion of the models throughout the registration process. Validation is performed on three tissue mimicking-phantoms designed to preserve realistic curvature of the spine. We compare pairwise and groupwise registration of the statistical shape model of the spine and demonstrate that clinically acceptable mean target error registration of $2.4 \mathrm{~mm}$ can be achieved with the proposed method. Registration results also show that the groupwise registration outperforms the pairwise in terms of success rate.

\subsection{Introduction}

Back pain is a major factor in decreasing the quality of life and productivity in North America [7]. In 2002, nearly two third of Canadian adults reported at least a mild level of back pain, leading to approximately $\$ 16.4$ billion in the cost of treatment and lost productivity [46]. In the United States, this cost has been reported as $\$ 62$ billion $[2,70]$. A more recent survey suggests these costs may be a lower estimate and real costs are in the range of $\$ 84.1$ to $\$ 624.8$ billion [18]. Oral medication is the standard primary method of treatment, however, [63] found that \%21 of patients reported no improvement and nearly half the patients surveyed reported little improvement in back pain. For these patients, this is simply not an effective form of treatment and the next step is spinal injections. Facet joint injections, a common percutaneous spinal injection procedure used for pain management, requires a high level of physician experience to effectively deliever the anesthetic dose to the target area. The success rate 
of proper needle insertion is a dismal $60 \%$ after 10 attempts [75] when no guidance is used, clearly an inadequate practice. Contemporary radiological needle guidance with CT and fluoroscopy requires specialized facilities often unavailable to patients living in rural areas, and involve X-ray radiation. In search of a more accessible, portable, and non-ionizing imaging alternative, ultrasound guidance has been considered. For facet joint injections, Watson et al. [74] and Klocke et al. [39] targeted the L3-L4 and L2-L3 interspace, a challenging procedure that, if performed inaccurately, damages the spinal cord. The results presented in both papers suggested that ultrasound as a solo guidance is inadequate. To address this issue, combination of ultrasound with CT has been proposed in an inter-modality registration framework [22, 55, 79].

Image registration is a key component in integrating the preoperatvie $\mathrm{CT}$ with intraoperative ultrasound. Previously in the literature, this inter-modality registration has been used for a wide variety of diagnostic [32] and therapeutic purposes, including: orthopedic procedures [5, 54], neurosurgical interventions [24], abdominal procedures [45] and cardiovascular operations [34, 38]. However, correct registration between ultrasound and CT images has proven to be a challenging problem [34, 38], because of the low Signal to Noise Ratio (SNR) of ultrasound images, the presence of US imaging artifacts in US and the fact that the same anatomy can appear quite differently in CT and ultrasound data. For this reason, most of the existing algorithms preprocess either of the modalities to reduce the artifacts and to increase the similarities in either images. For example, Roche et al. [65] register 3D US to MRI by using both intensity and gradient information. Similarly, Leroy et al. [45] register CT with freehand 3D US by preprocessing the CT images to boost edges and the US images to remove the speckle noise and shadows. Pre-operative image gradient/edge 
information has been also used by Pluim et al. [61]. In Huang et al. [34], both modalities are preprocessed to preserve only features which appear similar in either images. Penney et al. [60] remap both MRI and freehand 3D US to a probabilistic representation before co-registering. Recently, Wein et al. [76] has proposed to dynamically simulate ultrasound images from CT data based on the physics of image formation. The simulated image is updated throughout the registration process until a correct alignment is achieved between the two modalities.

For pain management, introducing ultrasound for imaging guidance removes the need for an intra-operative CT and fluoroscopy, which in turn greatly reduces the amount of ionizing radiation. As mentioned in the chapter 2, many papers have investigated SSMs as an alternative to preoperative CT scans to further reduce the ionizing radiation.

Our contribution is the first report of a groupwise SSM to 3D ultrasound registration without prior segmentation or preprocessing in the ultrasound data. To create the SSMs from individual vertebrae, we use a simple but generic approach based on the B-spline deformable transformation that allows for straightforward extension for an ensemble of vertebrae and relevant structures [4, 25, 35]. Furthermore, our algorithm lends itself to parallel implementation on the GPU, which rapidly decreases the run-time, thus allowing a faster convergence. 


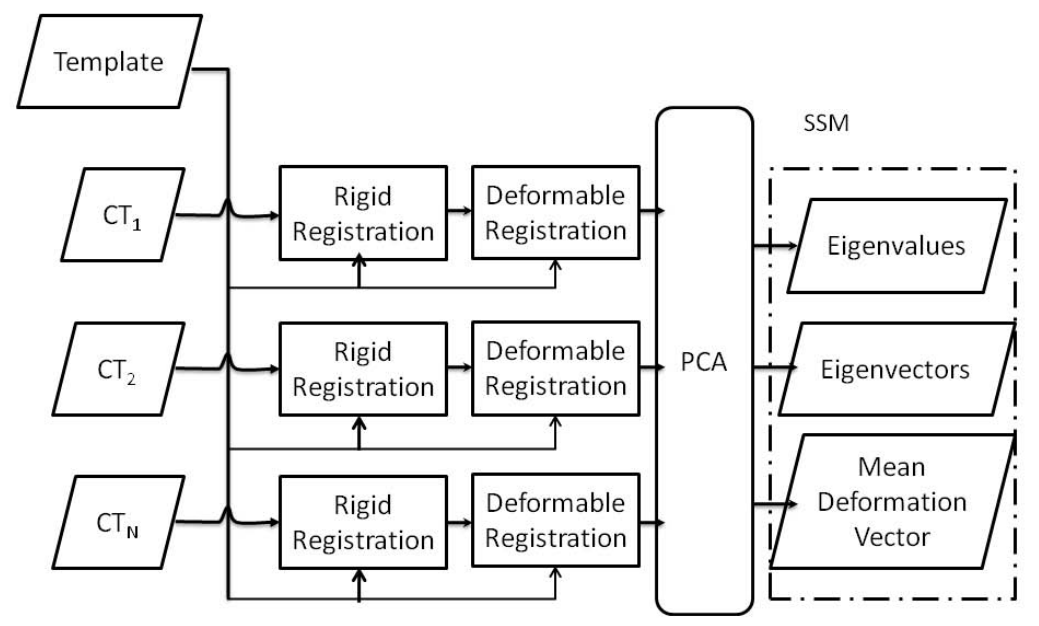

Figure 4.1: Outline of the SSM construction method from a set of CT images.

\subsection{Methods}

\subsubsection{Statistical Shape Model Construction}

Figure 4.1 demonstrates the SSM construction process for each vertebra. Initially, one of the $\mathrm{CT}$ volumes is chosen as the template, $I_{\mathrm{t}}$. Each training example, $I_{k}$ (from patient $C T_{k}$ ), is registered to the template by a rigid registration followed by a B-

spline deformable registration, such that $I_{\mathrm{t}} \approx T_{\text {def }}^{k}\left(T_{\text {rigid }}^{k}\left(I_{k}\right)\right)$, where $T($.$) denotes a$ transform. B-spline registration is performed in a $40 \times 30 \times 30$ grid using the mutual information metric. To reduce the deformable registration time, a three stage multiresolution approach is implemented. Each deformable registration takes between two and four hours on a Core 2 Quad CPU machine with $2.4 \mathrm{GHz}$ speed and $3 \mathrm{~GB}$ of RAM. With the deformable transform of all the training examples known with respect to the template, principal component analysis (PCA) is performed to construct the SSM. 
After the SSM is constructed, it can be used to generate new instances of the population. A new instance of the SSM, defined by the deformation vector, $D_{n e w}$, can be produced by a linear combination of the mean deformation vector, $\bar{\phi}$, SSM weights, $w_{i}$, and the eigenvectors of the covariance matrix generated from all the deformation fields, $v_{i}$ :

$$
D_{\text {new }}=\bar{\phi}+\sum_{i=1}^{N} w_{i} v_{i} .
$$

The SSM weights in equation (4.1) provide a compact description of the transformation needed to deform the mean shape into the shape of the new instance. Finding the number of eigenvectors needed to span the shape variations in the patient population is usually a tedious task. As a rule of thumb, the number of eigenvectors, $M$, needed to adequately represent the SSM population, should satisfy the following:

$$
\epsilon \geq \frac{\sum_{i=1}^{M} \lambda_{i}^{2}}{\sum_{i=1}^{N} \lambda_{i}^{2}}
$$

where $\lambda_{\mathrm{i}}$ are the eigenvalues in descending order, $N$ is the total number of eigenvalues and $\epsilon$ is a user defined threshold. In our case, the first 12 eigenvectors covered $95 \%$ of variations in shape.

\subsubsection{Pairwise Registration}

It is noted that throughout this text the pose is used as a synonym for the six rigid transform parameters (three for translation and three for rotation), which denote the position and orientation of the vertebra. Also the shape stands for the twelve eigenvectors, which correspond to the SSM weights using equation 4.1. In this section, we describe our proposed method for single-vertebra SSM to ultrasound registration. 
Solving the SSM to ultrasound registration problem, involves finding the best rigid and SSM weight parameters. Unfortunately, local extrema make this a challenging task. In order to reduce the complexity of the problem, we decouple the rigid and deformable registrations as shown in Figure 4.2. Starting from an initial rigid pose and zero SSM weights, first a rigid registration is performed to optimize the position and orientation of the mean shape. Then, starting from this pose, a deformable registration solves for the optimum shape and updates the SSM weights. The updated rigid and SSM parameters initialize the second iteration. This process is continued until the rigid and SSM weights converge.

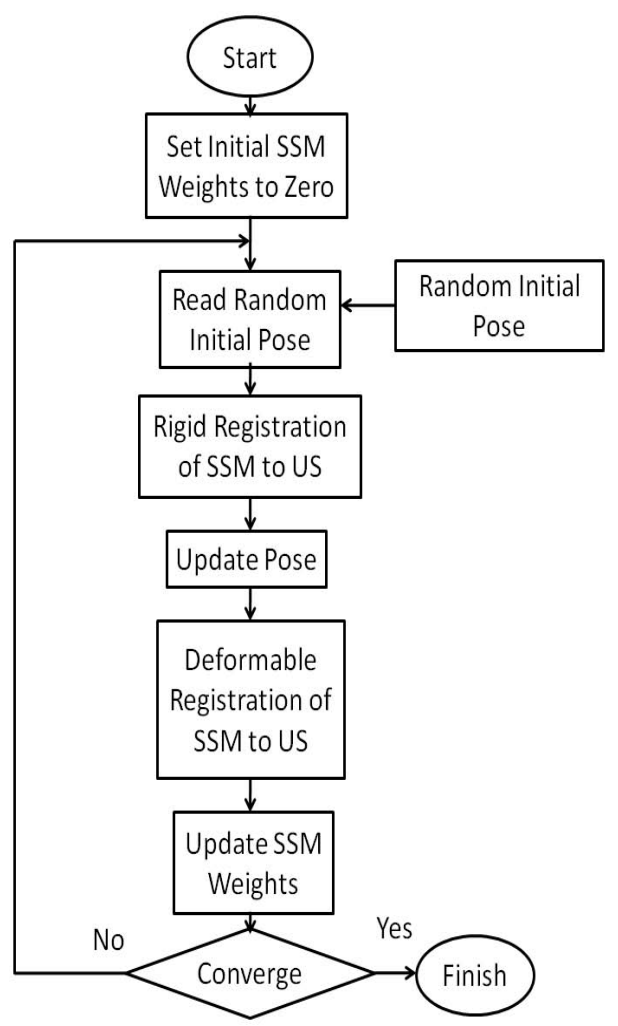

Figure 4.2: Registration workflow. 
The rigid and deformable registration blocks are illustrated in Figure 4.3. For the rigid registration block, Given the SSM and ultrasound as input, with the initial SSM weights set to zero, first an instance of the SSM is generated. Using a random initial six-degree-of-freedom rigid transform, the generated instance is brought to an initial alignment with the ultrasound volume. At this point, similar to [22, 76], an ultrasound volume is simulated from the instance, and compared with the patient ultrasound using a similarity metric. This similarity metric is passed to the optimizer to update the rigid transform parameters. A similar process is performed in the deformable registration block, only in this case the SSM weights are updated.

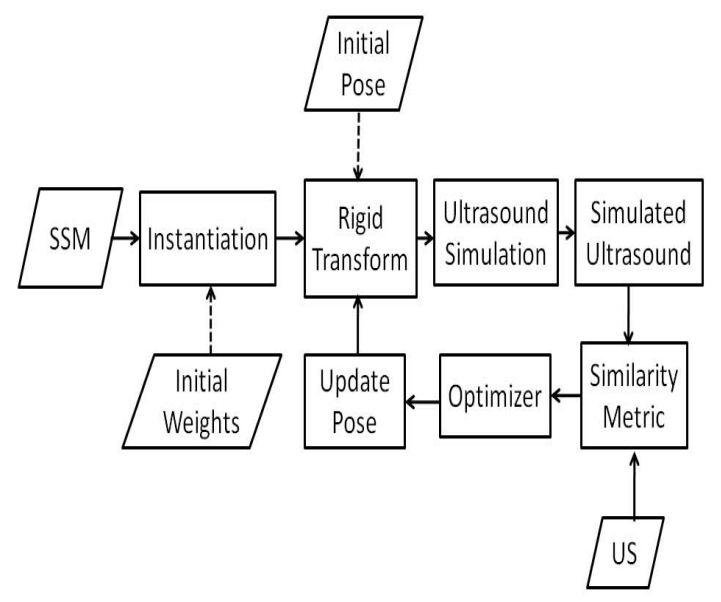

(a)

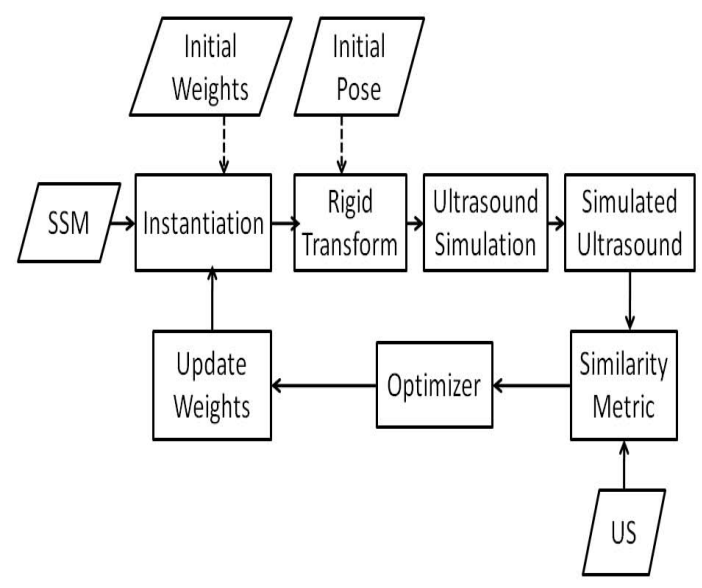

(b)

Figure 4.3: Detailed Description of the (a) Rigid and (b) Deformable Registration Blocks for Pairwise Registration

There are three steps in the simulation of ultrasound from CT: The simulation of the ultrasound reflection from CT, the mapping of the CT values to those found in ultrasound, and the calculation of weights for these two images and a bias. The weights are chosen so that the simulation best represents the real ultrasound image. 
The ray casting algorithm models the ultrasound reflections using an ultrasound beam traveling down each column of the CT volume. The implicit assumption in this case is that the CT intensities (in Hounsfield units) can be related to the acoustic impedance values used to calculate ultrasound transmission and reflection. The transmission and reflection of the beam is calculated at each voxel based on the following equations [76]:

$$
\begin{gathered}
\Delta r(x, y, d)=\left(d^{T} \nabla \mu(x, y)\right) \frac{\nabla \mu(x, y)}{2 \mu(x, y)} \\
\Delta t(x, y)=\left(1-\left(\frac{|\nabla \mu(x, y)|}{2 \mu(x, y)}\right)^{2}\right. \\
r(x, y)=I(x, y-1) \Delta r(x, y, d) \\
I(x, y)= \begin{cases}I(x, y-1) \Delta t(x, y) & \nabla \mu(x, y) \geq \tau, \\
0 & \nabla \mu(x, y)<\tau .\end{cases}
\end{gathered}
$$

where $d$ is the direction of the ultrasound beam, $\mu$ is the intensity of the CT image, $\Delta \mathrm{r}$ is reflection coefficient, $r$ is the simulated reflection intensity, $\Delta t$ is the transmission coefficient, $\tau$ is the threshold for full reflection, and $I$ is the intensity of our simulated ultrasound beam. Similar to [22], we assume that any gradient value greater than a set threshold (450 Hounsfield units in our simulations) represents the boundary of bony anatomy with the soft tissue and causes full reflection of the ultrasound beam intensity at that point, setting the incoming ultrasound beam intensity for all subsequent points on the scan line to zero. To amplify small reflections, a logcompression is applied to the simulated reflection image:

$$
r(x, y)=\frac{\log (1+\operatorname{ar}(x, y)}{\log (1+a)} .
$$


The CT intensities are mapped to values closer to those corresponding to the tissues in the ultrasound data using an approximation of the curve presented in [76]:

$$
p(x, y)=1.36 \mu(x, y)-1429 .
$$

The final step of the ultrasound simulation is the weighting of the simulated ultrasound reflection, the mapped CT and a bias term. Using a least-squares optimization technique to calculate the weights, the values are set as to best match the corresponding intensities in the real ultrasound volume. The final simulation is calculated as

$$
f(x, y)= \begin{cases}\alpha p(x, y)+\beta r(x, y)+\gamma & I(x, y)>0 \\ 0 & I(x, y)=0\end{cases}
$$

where $f$ is the simulated ultrasound image, and $\alpha, \beta$ and $\gamma$ are the weights for their respective images. We do not include any voxels that are occluded in the simulation as part of the weight calculation. All occluded voxels in the ultrasound simulation are set to zero. Occluded voxels are identified as any voxel where the intensity of the incoming simulated ultrasound beam is zero. Similarity between the actual ultrasound image, $U$, and simulated ultrasound image is calculated using the Linear Correlation of Linear Combinations $\left(\mathrm{LC}^{2}\right)$ metric presented by [76]:

$$
L C^{2}=\frac{\sum(U(x, y)-f(x, y))^{2}}{M \times \operatorname{Var}(U)},
$$

where $N$ is the number of overlapping voxels between the ultrasound and CT images. All voxels, including occluded voxels, are used in the calculation of the similarity metric. We use the Covariance Matrix Adaptation Evolution Strategy (CMA-ES) [30] as the optimizer. 


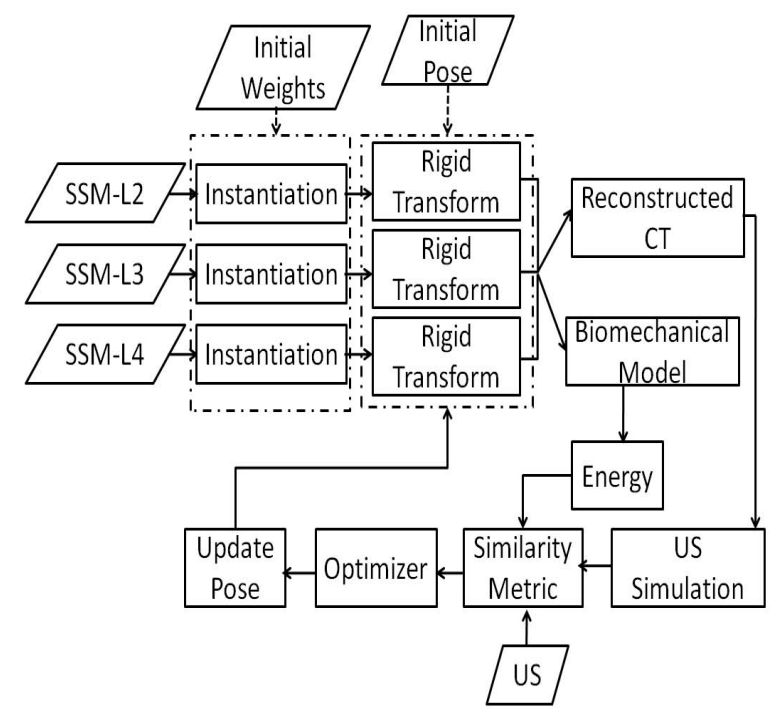

(a)

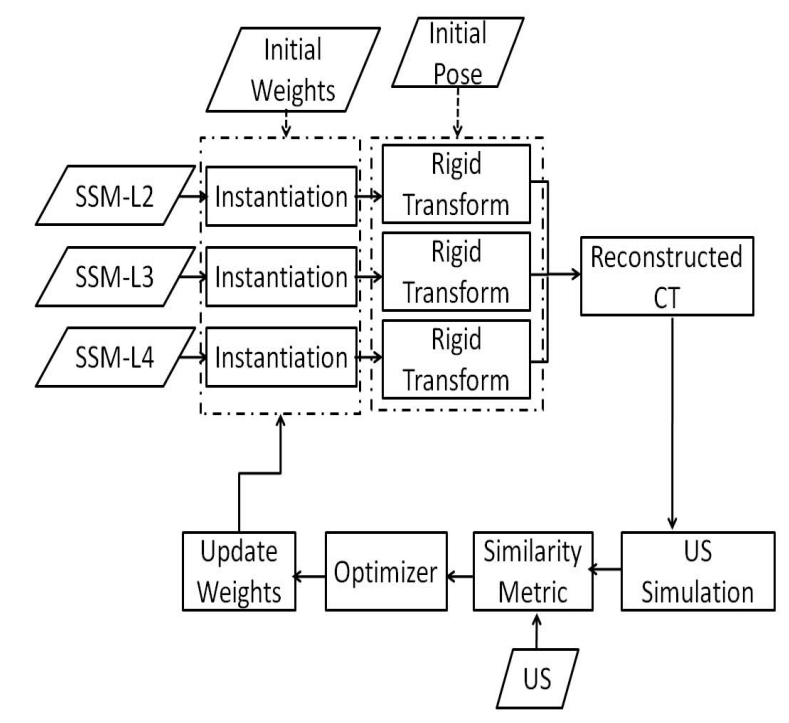

(b)

Figure 4.4: Detailed Description of the (a) Rigid and (b) Deformable Registration Blocks for Groupwise Registration

\subsubsection{Groupwise Registration}

Similar to the pairwise registration, as shown in Figure 4.2, we have split the rigid and deformable groupwise registration problems into two separate blocks. In our approach, each vertebra is treated individually. Thus, solving a three vertebrae groupwise registration problem will involve optimizing for 36 SSMs weights (12 for each vertebra) and 18 rigid parameters (six for each vertebra) to find the correct shape and pose. Decoupling the problem, into rigid and deformable registration blocks, not only decreases the complexity of the problem, but also allows for a faster convergence by decreasing the population size of the CMA-ES optimizer.

Figure 4.4(a) illustrates the groupwise rigid registration block. First, setting the initial SSM weights to zero, an instance of each L2, L3 and L4 vertebrae is generated. Then, using a random initial rigid transform, each generated instance is perturbed to 
an initial pose. After the rigid transforms are applied, the instances are reconstructed into a single volume. For any overlapping voxels, the maximum intensity is selected for the reconstructed volume, thus preserving bone structure. Any gaps not in the final volume are filled with a default value approximating the intensity of soft tissue in CT. The ultrasound simulation is applied to the reconstructed volume. As the next step, the $\mathrm{LC}^{2}$ metric is calculated and is fused with the biomechanical model to produce the final similarity measure. This similarity measure is fed to the CMA-ES optimizer until all of rigid parameters converge. An analogous approach is employed for the groupwise deformable registration block, as shown in Figure 4.4(b).

\subsubsection{Biomechanical Model}

Allowing free motion of the vertebrae during the course of the groupwise registration may result in anatomically unrealistic alignments where the vertebrae are in invalid orientations, colliding or far apart. In order to constrain the space of possible registration outcomes, a biomechanical model is employed [22]. This model relates the relative displacements of vertebrae with the reaction forces and moments using the stiffness matrix, $K$, proposed in $[22,19]$. As an approximation, the vertebrae are expected to remain in a common rotational orientation with no translation perpendicular to the sagital and coronal planes. The expected translation perpendicular to the axial plane is used as an approximation of the mean distance between neighboring vertebrae. The energy of the system is given using the spring equation:

$$
U=\frac{1}{2}\left(x^{T} K x\right)
$$


where $K$ is the stiffness Matrix, measured in N.mm.rad ${ }^{-1}$, as proposed in [19]:

$$
\begin{gathered}
K= \\
{\left[\begin{array}{cccccc}
100 & 0 & 50 & 0 & -1640 & 0 \\
0 & 110 & 0 & 150 & 0 & 580 \\
50 & 0 & 780 & 0 & -760 & 0 \\
0 & 150 & 0 & 1.48 E 5 & 0 & -8040 \\
-1640 & 0 & -760 & 0 & 1.52 E 5 & 0 \\
0 & 580 & 0 & -8040 & 0 & 1.53 E 5
\end{array}\right]}
\end{gathered}
$$

The total energy is calculated over all the vertebrae and normalized with respect to the maximum misalignment energy, which in our case, is generated by $10 \mathrm{~mm}$ translation along the axes and 10 degrees about each axis:

$$
E=\frac{U_{L 2, L 3}+U_{L 3, L 4}}{2 \times U_{\max }}
$$

The normalized energy is blended in the computation of Biomechanically Constrained Linear Correlation of Linear Combinations as suggested by Gill et al. [23]:

$$
B C L C^{2}=L C^{2}-\sigma E
$$

where $\sigma$ is a constant that determines the strictness of the biomechanical model. A value of zero for the strictness would remove the biomechanical model entirely, whereas large values for the strictness would fix the relative position and orientation of the vertebrae throughout the registration. In our case, we used a value of 0.75 as Gill et al. [22] found it to be the optimum strictness constant in terms of registration success rate. 


\begin{tabular}{cccc}
\hline SSM & $\begin{array}{c}\text { Average CPU Time } \\
\text { sec }\end{array}$ & $\begin{array}{c}\text { Average GPU Time } \\
\text { msec }\end{array}$ & Gain \\
\hline L2 & 2.3 & 8.4 & 273 \\
L3 & 2.4 & 7.2 & 342 \\
L4 & 2.5 & 8.2 & 308
\end{tabular}

Table 4.1: CPU vs. GPU Instantiation run-times and the performance gain computed as CPU/GPU. A total of 100 speed tests are performed on the CPU and the GPU. In each test, a random combination of SSM weights is passed to the instantiation block.

\subsubsection{Parallel Implementation on Graphics Processing Unit}

Running the proposed registration algorithm on a desktop computer, will take close to a day for a pairwise registration and a week for groupwise registration, rendering it useless for real-time clinical applications. To speed up the registration, we have implemented a parallel version of our registration algorithm using CUDA on a nVIDIA GTX 285 GPU with 2 GB of RAM and 240 cores, each running on a $648 \mathrm{MHz}$ graphics clock.

The bottle-necks in our registration algorithm are the SSM instantiation and ultrasound simulation. Details of the GPU-accelerated SSM instantiation technique and ultrasound simulation have been reported in [27] and [12] respectively. Also a brief description of the parallel implementation on the GPU is given in Appendix A. For the specific spine data sets used in this manuscript, as shown in Table 4.1, using GPU, a speed gain of close to $300 \times$ can be achieved for SSM instant generation. The GPU-accelerated ultrasound simulation implementation can achieve a speed gain of $70 \times$ in our implementation. 


\subsection{Materials}

\subsubsection{CT Data Acquisition}
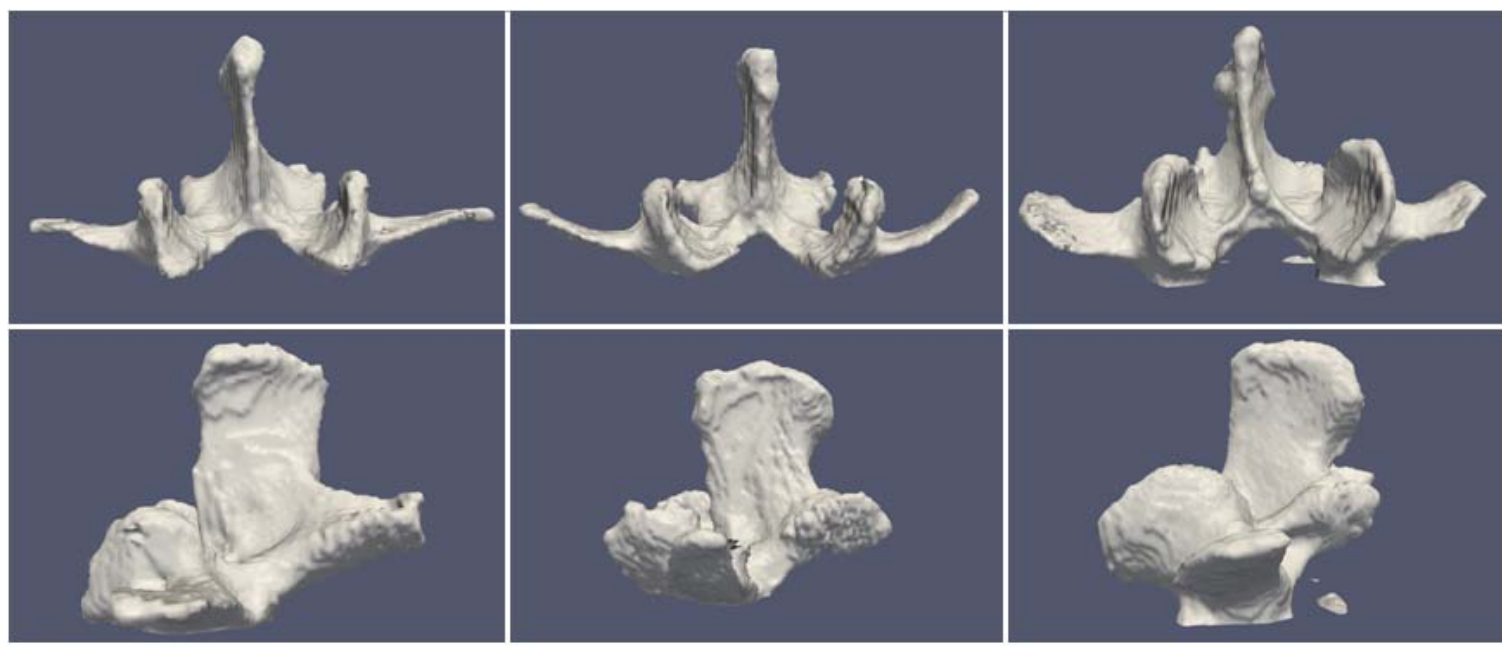

Figure 4.5: Samples of the segmented CT images acquired from the patients. Top: Axial view; bottom: Sagittal view

The CT data was collected at the Kingston General Hospital under the approval of the Research Ethics Board. The patients involved provided written consent for this data to be used in clinical studies. A set of CT images, acquired from 38 patients (19 male and 19 female), is used in this study. The L2, L3 and L4 vertebrae are semiautomatically segmented from patient CT images using ITK-Snap and resampled into $120 \times 200 \times 100$ volumes with an isotropic spacing of $0.6 \mathrm{~mm}$. The patient data is divided into two groups: 35 for constructing the SSM (hereafter referred to as training data), and 3 for validation (two male and one female). Samples of the patient CT data are shown in Figure 4.5. 


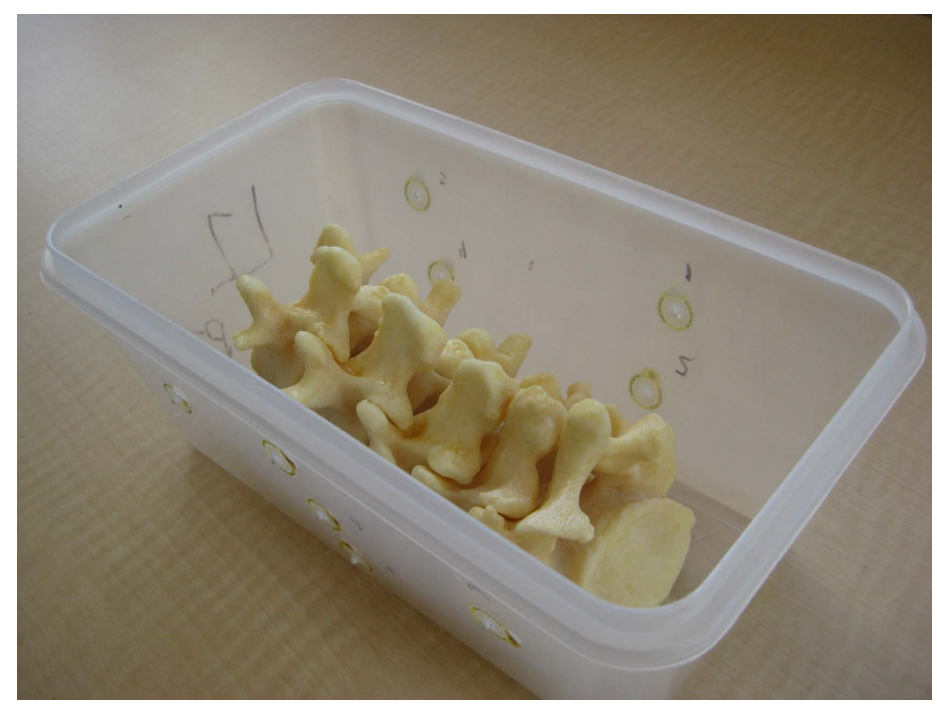

Figure 4.6: Printed phantom with a realistic spinal curvature. The fiducial markers on the exterior of the box register the acquired ultrasound to the corresponding CT volume.

\subsubsection{Ultrasound Data Acquisition}

The three excluded CT volumes from the patient data are used to construct 3D models of the entire lumbar spine, including L1 to L5. The L1 and L5 were intentionally printed as well, since in real patients, parts of the L2 and L4 vertebra are occluded in US images by the L1 and L5. These virtual models are transformed into physical models using a Cimetrix 3D shape printer (Cimetrix Solutions, Oshawa, ON, Canada) as in Figure 4.6. Three spine phantoms are constructed by submerging these physical models in an agargelatine-based tissue phantom which is designed to simulate the appearance of soft tissue in ultrasound [49]. The soft tissue is cooked according to the recipe shown in Table 4.2, and slowly poured in layers into the phantom box covering the spine model entirely. A high-resolution CT image $(0.46 \times 0.46 \times 0.625 \mathrm{~mm})$ 


\begin{tabular}{ccccccc}
\hline Chemicals & Agar & Gelatine & Cellulose & Glycerol & Germal Plus & Water \\
\hline Weight \% & 1.17 & 3.6 & 3 & 3.18 & 1 & 88.05 \\
\hline Weight (g) & 37.21 & 114.48 & 95.40 & 101.12 & 31.80 & 2800 \\
\hline
\end{tabular}

Table 4.2: Recipe for the phantom construction in Figure 4.6

and an ultrasound volume are acquired from each phantom. The ultrasound volume is reconstructed from a freehand sweep with an L14-5/38 linear-array transducer (Ultrasonix, Richmond, BC, Canada) operating at 6.6 MHz with an imaging depth of $5.5 \mathrm{~cm}$. The probe is tracked using an Optotrack Certus System (Northern Digital Inc., Waterloo, ON, Canada) and calibrated using an N-wire phantom [13]. The phantom CT and ultrasound volumes are aligned using fiducial markers mounted on the exterior of the phantom box. The position of these fiducials in the ultrasound coordinate system is identified using a calibrated stylus pointer.

\subsection{Experiments and Results}

\subsubsection{Registration of Statistical Shape Model to CT Data}

We initially investigate whether the statistical shape model generated from L3 can span the space of shape variations in the patient population. For this purpose, we use a leave-one-out cross registration with the $\mathrm{CT}$ data. In each step, we leave out one of the patient CTs for testing, create the SSM from the rest of the data, and register the resulting SSM to the remaining patient CT. This process is repeated by selecting each CT data once as the testing data. Throughout the process, the template is held 


\begin{tabular}{c|c|c}
\hline SSM & RMS $(\mathrm{mm})$ & STD $(\mathrm{mm})$ \\
\hline L2 & 0.95 & 0.23 \\
L3 & 0.89 & 0.19 \\
L4 & 0.80 & 0.33
\end{tabular}

Table 4.3: Results for the leave-one-out experiments for each vertebrae

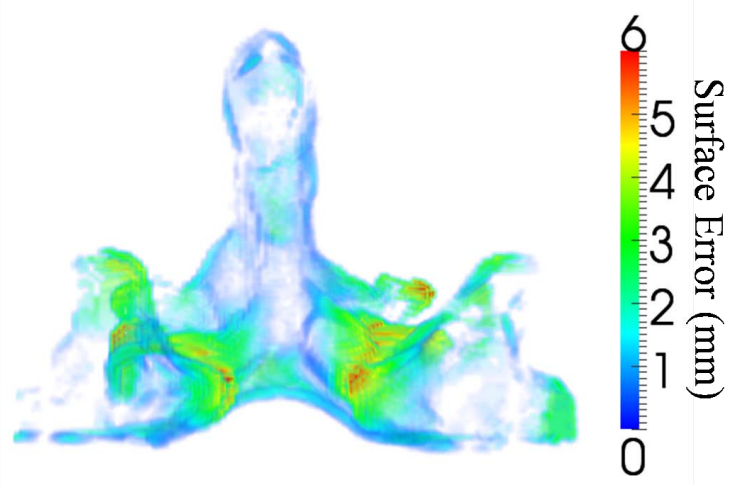

Figure 4.7: An example of the surface error distribution for SSM to CT registration on the L3 vertebra.

constant. To validate the model, we use the first 12 eigenvectors which cover $95 \%$ of the total modes of variation. The registration process uses mutual information and deformable B-Spline registration, in conjunction with the CMA-ES optimizer to solve for the instance of SSM that best fits the $\mathrm{CT}$ and the corresponding rigid registration parameters.

As in [72], the Root Mean Square (RMS) error between the closest surface points of registered SSM and the test data is used to measure the capability of the SSM to capture the deformations within the patient population. The mean error across all registrations for each vertebrae is shown in Table 4.3 and illustrated in Figure 4.7. 


\subsubsection{Pairwise Registration}

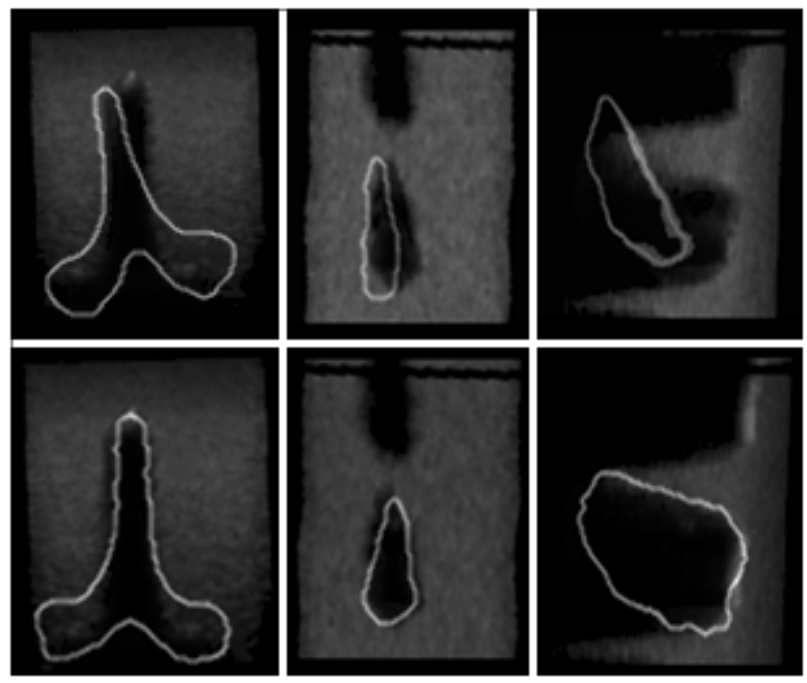

Figure 4.8: Transverse (left), coronal (center), and sagittal (right) slices of the original US volume overlayed with the bone contours of the misaligned (top) and pairwise registered (bottom) SSM volumes.

In a second set of experiments, we aim to show how well the constructed SSM can be registered to match the shape and position of the individual vertebrae using the acquired 3D ultrasound images. In each registration, as in Figure 4.2, we interchangeably optimize 12 shape and six rigid parameters, which correspond to the first 12 PCA eigenvectors and the pose, respectively.

The SSM mean shape and the ultrasound volumes are brought to an initial position by rigidly registering the mean shape to the corresponding phantom $\mathrm{CT}$ volume. For each phantom, 30 experiments are performed with perturbing the mean shape using a 
transformation generated from a uniform random distribution in the interval of $[0,10]$ mm translation along each axis and $[0-10]^{\circ}$ rotation about each axis. The registration parameters are then optimized as it is discussed in Section 4.3.2.

To evaluate the accuracy of the registration, an expert orthopedic surgeon is asked to identify five corresponding landmarks, three on the spinous process and two on the facet joints, as shown in Figure 4.9, on the registered SSM, the ultrasound volume and the corresponding CT. The average distance of these five landmarks are chosen as a measure of the final Target Registration Error (TRE). A registration is considered failed if the final TRE is more than $3.0 \mathrm{~mm}$, as the clinically accepted error. Registration results are shown in Table 4.4 and an example of the initial misalignment and the registration result is depicted in Figure 4.8. Figure 4.9 shows the overlay of the registered SSM with the ultrasound volume.

\begin{tabular}{c|c|c|c|c|c|c|c|c|c}
\hline \multirow{2}{*}{ Phantom } & \multicolumn{3}{|c|}{ L2 } & \multicolumn{3}{c|}{ L3 } & \multicolumn{3}{c}{ L4 } \\
\cline { 2 - 10 } & Mean & STD & SR & Mean & STD & SR & Mean & STD & SR \\
\hline 1 & 2.48 & 0.29 & $75 \%$ & 2.37 & 0.42 & $71 \%$ & 2.41 & 0.34 & $73 \%$ \\
2 & 2.36 & 0.43 & $70 \%$ & 2.56 & 0.33 & $69 \%$ & 2.41 & 0.45 & $69 \%$ \\
3 & 2.58 & 0.37 & $68 \%$ & 2.45 & 0.41 & $72 \%$ & 2.51 & 0.27 & $74 \%$ \\
\hline
\end{tabular}

Table 4.4: Results for the SSM to ultrasound pairwise registration. SR (Success Rate) is defined as the ratio of the registrations where the overall TRE is less than $3.0 \mathrm{~mm}$. SR is presented for each phantom with the maximum initial misalignment of $10 \mathrm{~mm}$.

\subsubsection{Groupwise Registration}

As the final step of the validation process, we intend to study the feasibility of recovering the shape, position and orientation of the L2, L3 and L4 vertebrae using the 


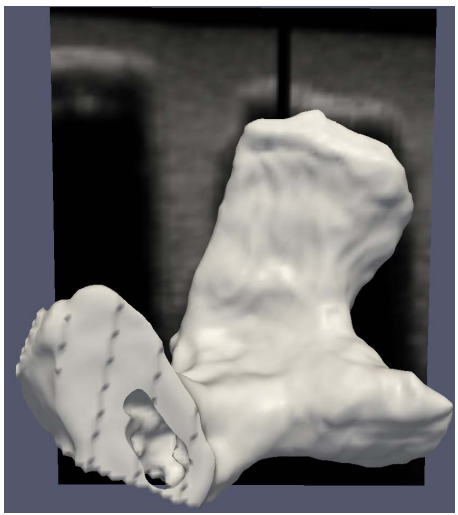

(a) L2-Sagittal

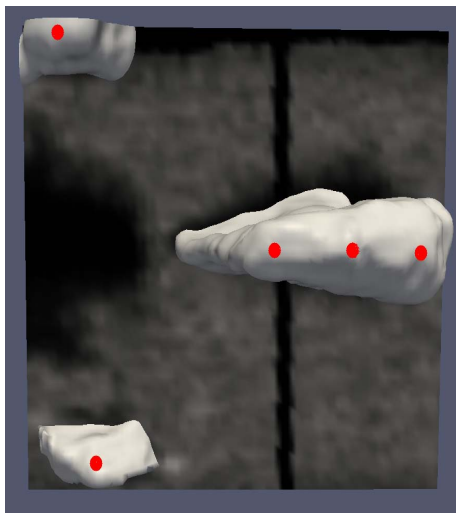

(d) L2-Coronal

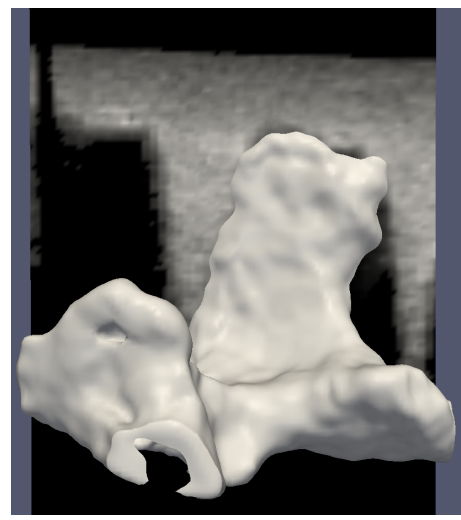

(b) L3-Sagittal

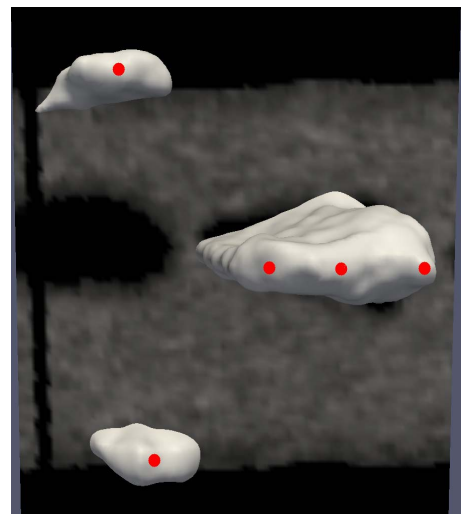

(e) L3-Coronal

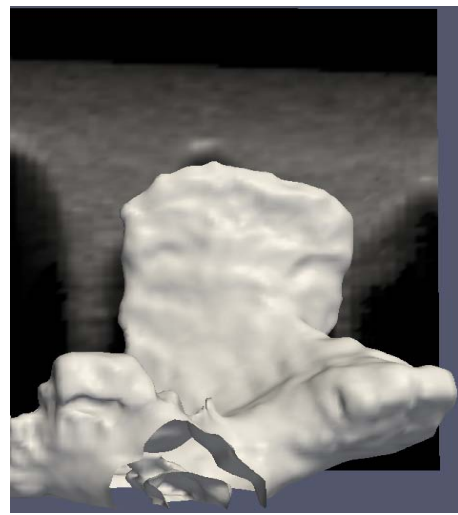

(c) L4-Sagittal

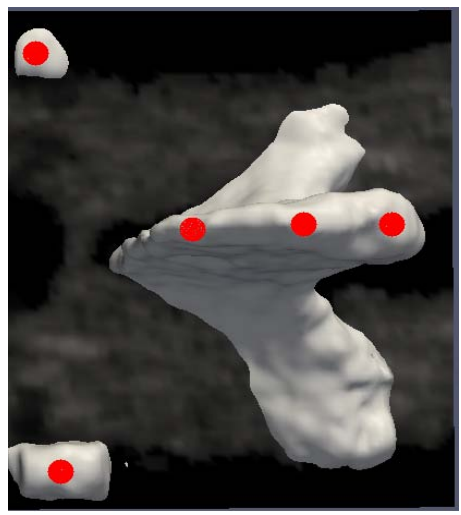

(f) L3-Coronal

Figure 4.9: Pairwise registration results for the L2-L4 vertebrae. The five red circles illustrate the landmark positions.

groupwise SSM to ultrasound registration framework. First, the SSMs of the L2, L3 and L4 are brought to an initial alignment by a rigid registration between the mean instance and the corresponding phantom $\mathrm{CT}$ volume. For each phantom, a total of thirty experiments are performed starting from a random position and orientation about the initial alignment. This random perturbation is generated using a uniform distribution in the $[0,10] \mathrm{mm}$ translation along each anatomical axis and $[0,10]^{\circ}$ rotation about each axis. In order to prevent inter-vertebral collision and unrealistic 

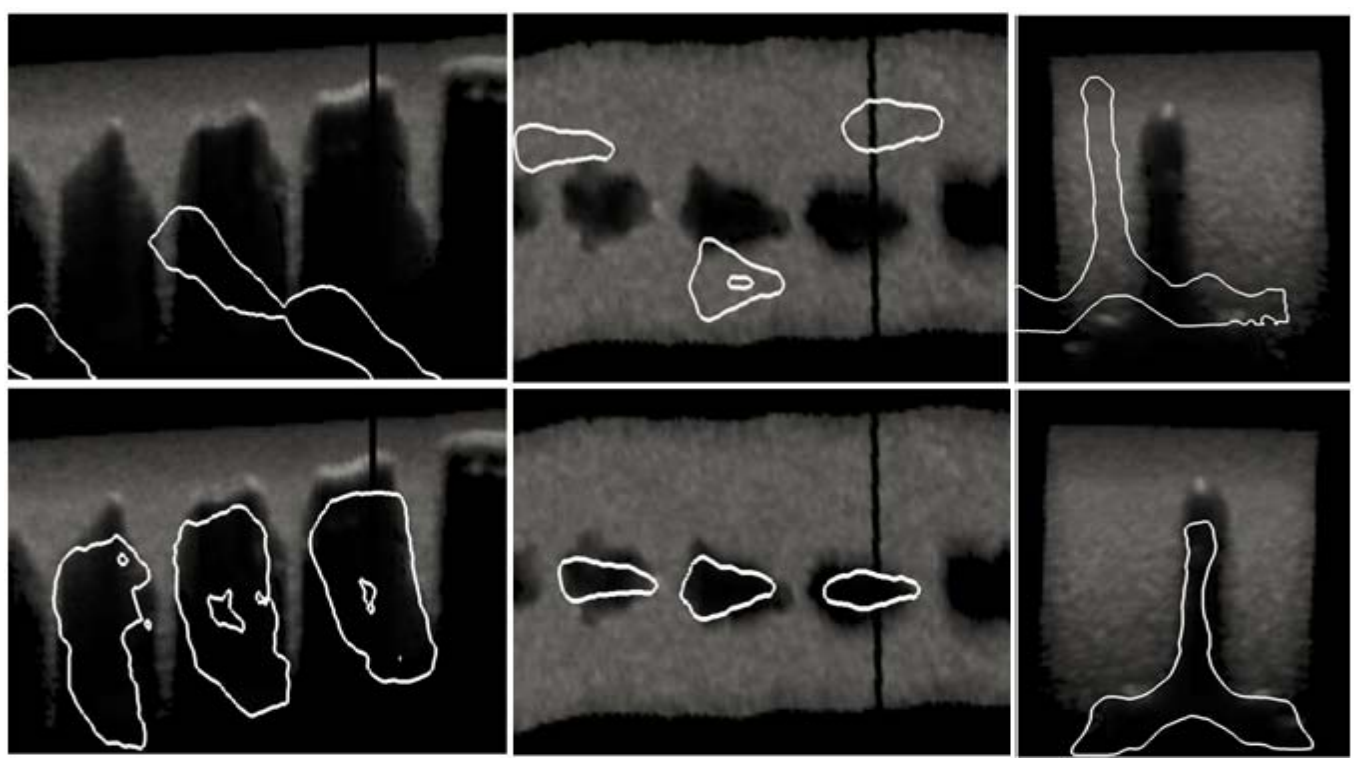

Figure 4.10: Transverse (left), coronal (center), and sagittal (right) slices of the original ultrasound volume overlayed with the bone contours of the misaligned (top) and groupwise registered (bottom) SSM volumes.

alignments, the biomechanical model is employed. To further reduce the registration time, instance generation and ultrasound simulation which are the computationally expensive parts of our algorithm, are parallelized in the GPU.

As in the pairwise case, an expert orthopedic surgeon is asked to identify 15 anatomical landmarks on the surface of the superior and inferior articular process of each vertebra on the registered SSM, the ultrasound and the corresponding CT volume, as illustrated in Figure 4.11. The average distance of the five landmarks are chosen as a measure of the final TRE. The groupwise registration is considered a success, only if the final TRE for each vertebra was less than $3.0 \mathrm{~mm}$. The success rate and the final TRE for each set of the experiments are shown in Table 4.5. Figure 4.10 shows the overlay of the registered SSMs of L2,L3 and L4 with the ultrasound volume. 


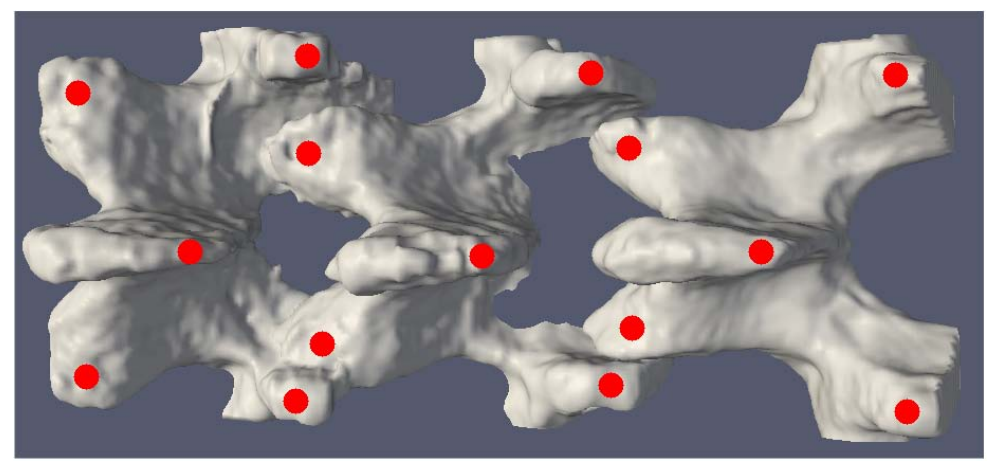

Figure 4.11: The red circles illustrate the position of the surgeon-selected landmarks on the surface of the vertebrae.

\subsection{Discussion}

Comparing the results in Table 4.5 and Table 4.4 shows that the success rate for L3 in the groupwise technique is higher in comparison to the pairwise approach. This is expected since in the case of L3, the neighboring vertebrae (L2 and L4) constrain the set of solutions for the registration outcome. Furthermore, as seen in Tables 4.4 and 4.5, both pairwise and groupwise registration methods lead to an average success rate of $70 \%$ with the average TRE below $3 \mathrm{~mm}$ through out the successful cases.

In order to investigate the statistical significance of the groupwise registration in comparison to the pairwise, an unpaired t-test was performed on the registration results, with the null hypothesis being that the two control groups share a common mean. We used the outcome of 30 registration trials to compare the results for the pairwise and groupwise approaches. The results, shown in Table 4.6, shows that the pairwise and groupwise registration results fail to show statistically significant difference.

The landmark errors for successful cases are below $3 \mathrm{~mm}$ which is a sufficiently 


\begin{tabular}{c|c|c|c|c|c|c|c|c|c|c}
\hline \multirow{2}{*}{ Phantom } & \multicolumn{3}{|c|}{ L2 } & \multicolumn{3}{c|}{ L3 } & \multicolumn{3}{c|}{ L4 } & \multirow{2}{*}{} \\
\cline { 2 - 10 } & Mean & STD & SR & Mean & STD & SR & Mean & STD & SR & GSR \\
\hline 1 & 2.49 & 0.49 & $79 \%$ & 2.56 & 0.33 & $82 \%$ & 2.36 & 0.43 & $79 \%$ & $79 \%$ \\
2 & 2.40 & 0.41 & $70 \%$ & 2.41 & 0.29 & $75 \%$ & 2.36 & 0.43 & $70 \%$ & $70 \%$ \\
3 & 2.43 & 0.36 & $75 \%$ & 2.4 & 0.34 & $82 \%$ & 2.56 & 0.33 & $75 \%$ & $75 \%$ \\
\hline
\end{tabular}

Table 4.5: Results for the SSM to ultrasound group-wise registration. Success Rate (SR) is defined as the ratio of successful results over the total number of registrations. Individual success rate for each vertebra and also the Group Success Rate (GSR) for all vertebrae are reported. A registration is considered a success (GSR) only if the final TRE is less than $3.0 \mathrm{~mm}$ for each vertebra.

\begin{tabular}{c|c|c|c}
\hline Phantom & L2 & L3 & L4 \\
\hline 1 & 0.86 & 0.53 & 0.56 \\
2 & 0.58 & 0.42 & 0.29 \\
3 & 0.64 & 0.40 & 0.33
\end{tabular}

Table 4.6: T-test probability values for comparing pairwise vs. groupwise registration

close distance to envelope the nerve sack in anesthetic agent without endangering the spinal cord. Results also demonstrate the feasibility of using volumetric SSMs for the registration of a patient spine to 3D ultrasound data. This can enable the use of ultrasound image guidance for spinal interventions without prior CT, to improve the efficacy of these procedures in an affordable manner.

\subsection{Conclusion}

We have demonstrated the technical feasibility of groupwise registration of intraoperative 3D ultrasound to volumetric statistical shape models of the lumbar spine, and laid the groundwork for subsequent clinical experiments. To the best of our 
knowledge, this is the first report of a groupwise SSM to ultrasound registration for spinal anatomical structures. The proposed technique has the potential to substantially improve existing image guided spinal injections, by substituting ultrasound as the sole imaging modality and thus removing expensive and ionizing intra-operative $\mathrm{CT}$ and fluoroscopy images.

In this work, we were limited to 35 patients for the construction of the SSM. While better results are expected with the increase of the number of patients in the training phase, a more quantitative approach in choosing the sample size will yield to a more optimum SSM training as suggested by Mei et al [52].

Future research will be to validate the registration technique on patient ultrasound data. Since the main purpose of this SSM to ultrasound registration is to assist in guiding needle placement in percutaneous spinal interventions, a user study in a clinical setting must be performed and is the subject of our current efforts. 


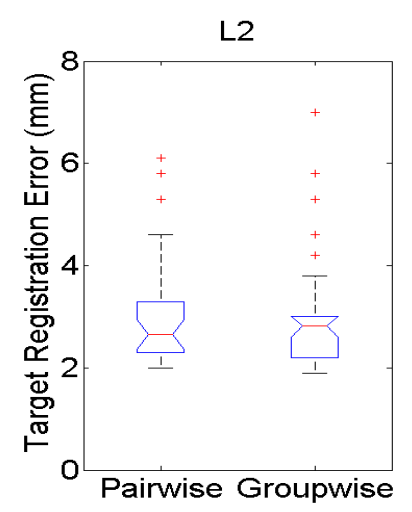

(a)

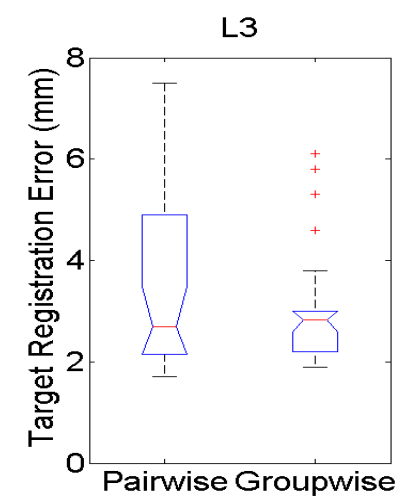

(d)

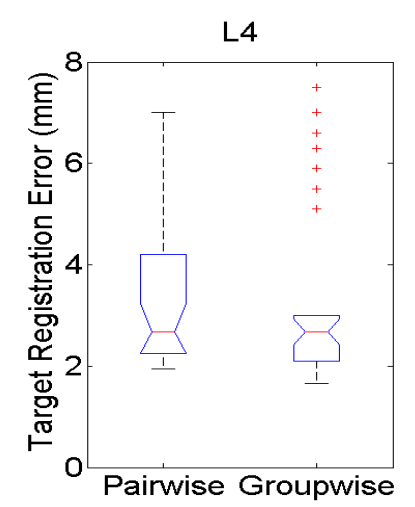

(g)

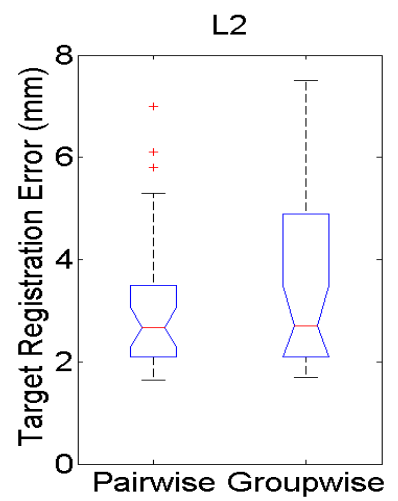

(b)

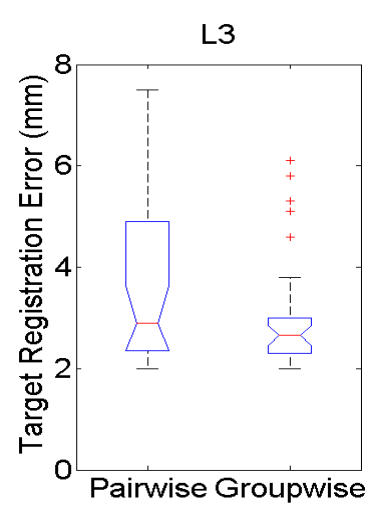

(e)

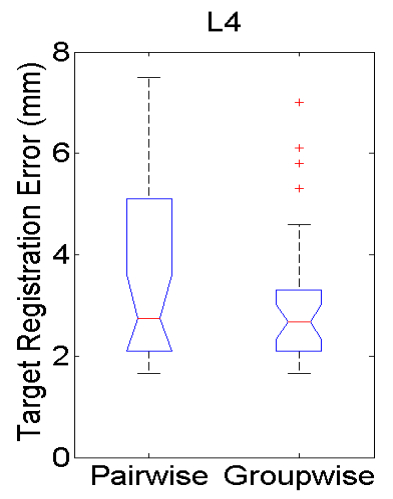

(h)

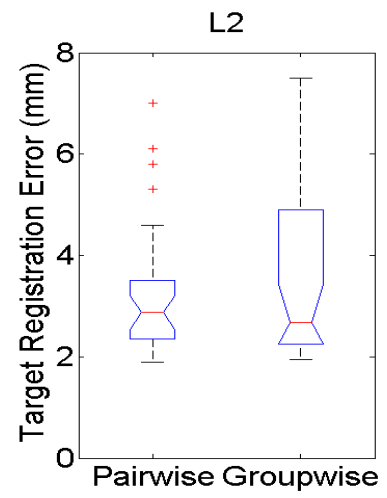

(c)

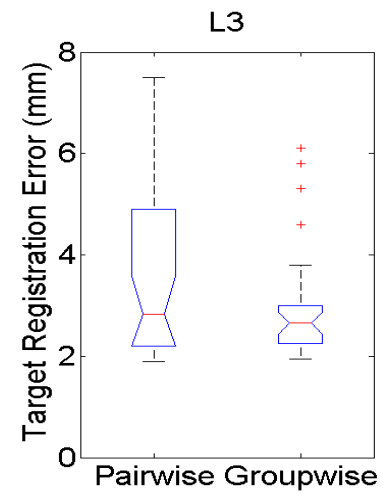

(f)

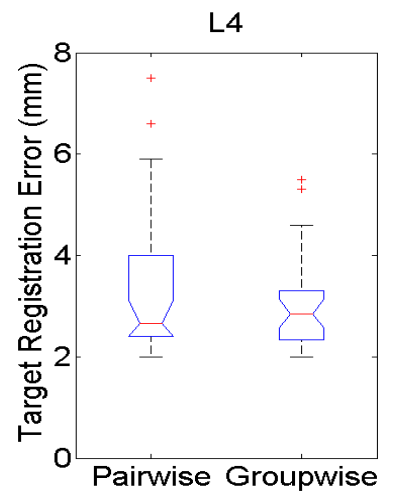

(i)

Figure 4.12: Graphical representation of the registration results for pairwise and groupwise registration. Phantoms 1, 2 and 3 (left to right) and the L2, L3 and L4 (top to bottom). 


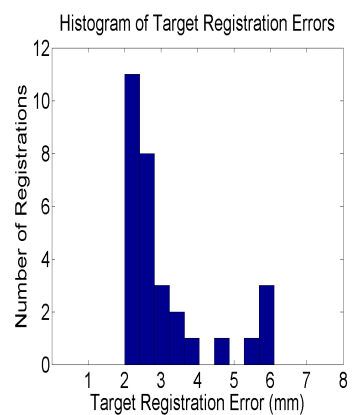

(a)

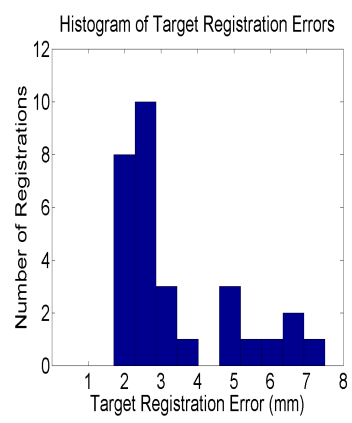

(d)

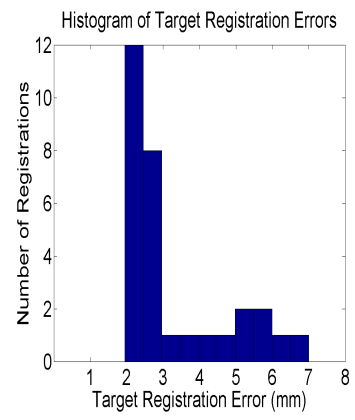

(g)

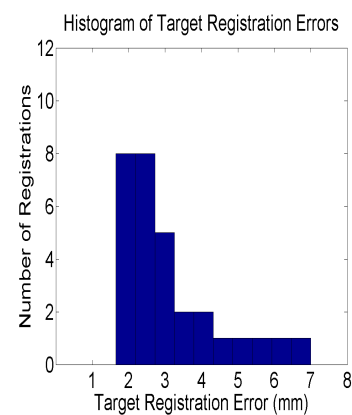

(b)

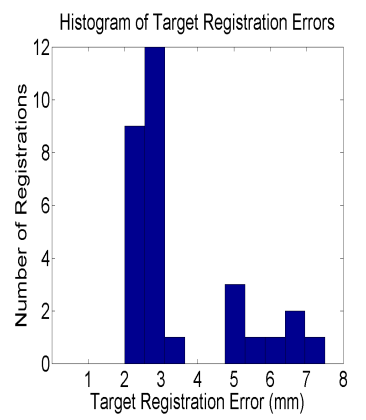

(e)

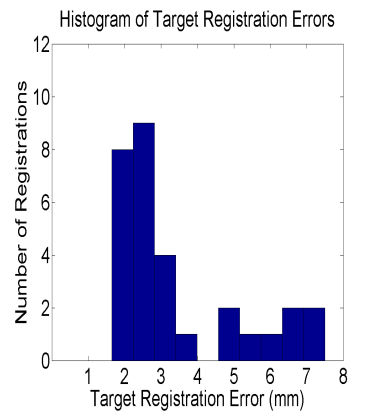

(h)

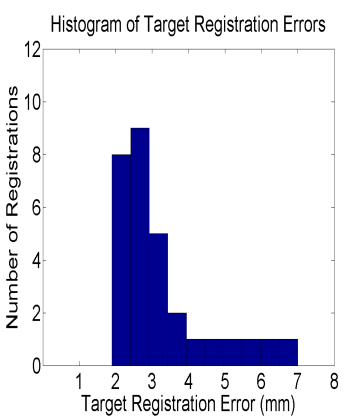

(c)

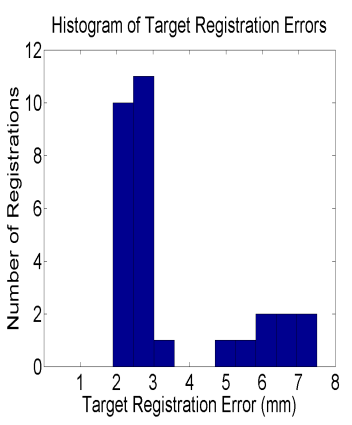

(f)

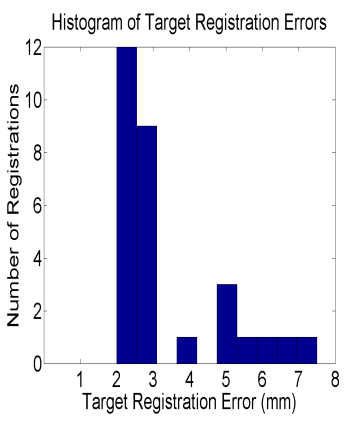

(i)

Figure 4.13: Histogram for target registration errors for the pairwise registration. Phantoms 1, 2 and 3 (left to right) and the L2, L3 and L4 (top to bottom). 


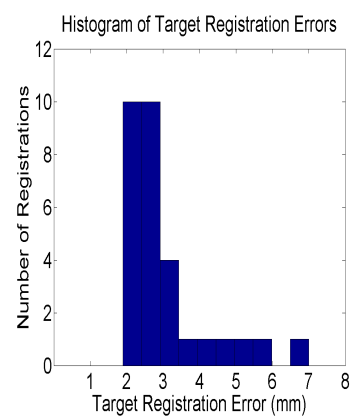

(a)

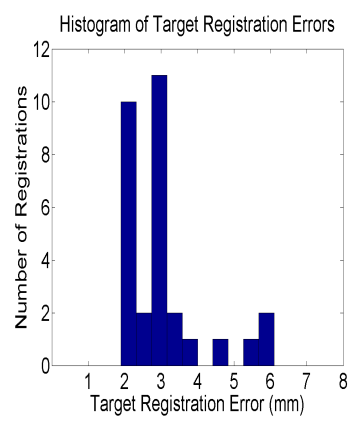

(d)

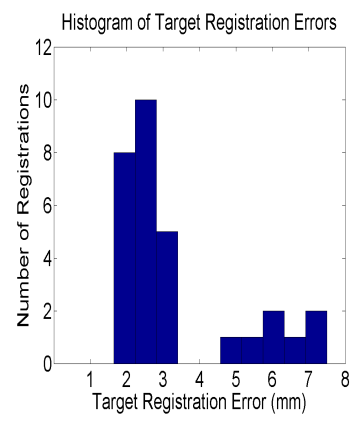

(g)

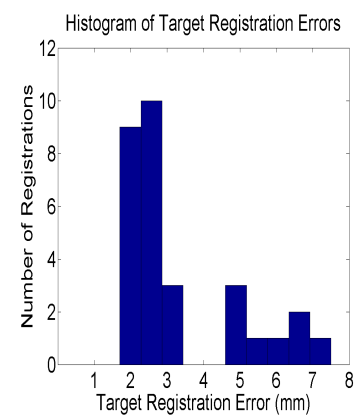

(b)

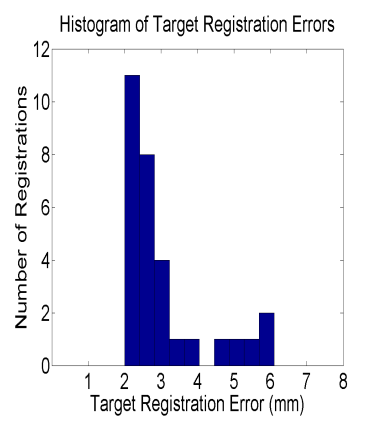

(e)

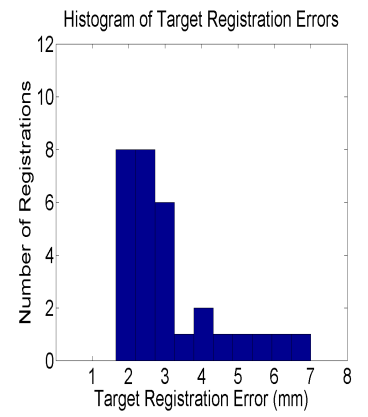

(h)

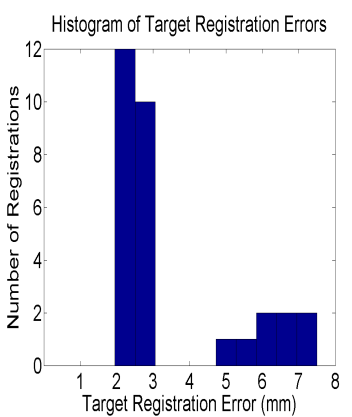

(c)

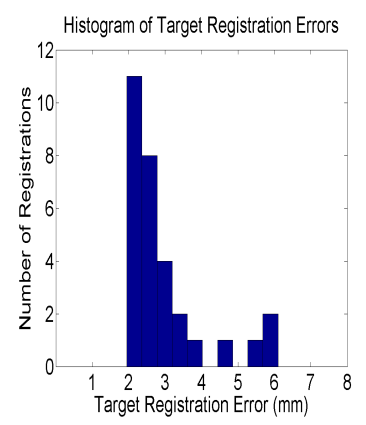

(f)

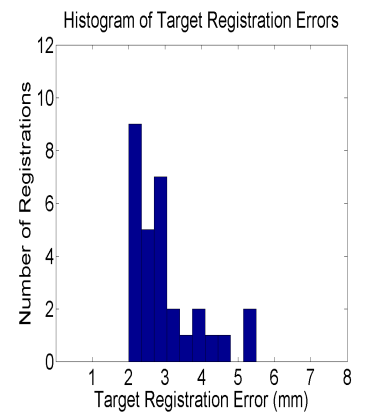

(i)

Figure 4.14: Histogram for target registration errors for the groupwise registration. Phantoms 1, 2 and 3 (left to right) and the L2, L3 and L4 (top to bottom). 


\section{Chapter 5}

\section{Conclusion and Future Work}

This chapter summarizes the contributions and results of this thesis. It then discusses the future work to improve the proposed system and its further validation in a clinical setting.

\subsection{Summary of Contributions}

In this thesis, a novel groupwise US to SSM registration technique for the lumbar spine is introduced which not only retrieves the position and orientation of the SSMs, but it also finds the shape of the SSMs. This is intended for the fusion of intraoperative US and prior knowledge on spinal anatomy, and is to be used for guidance in percutaneous spinal injections. The algorithm is designed with the following features:

1. The constructed SSMs are a sufficient representation of the L2-L4 vertebrae and can effectively retrieve the volumes in the training data. Furthermore, the constructed SSMs can be used to approximately represent volumes that have not been included in the training data. 
2. The US simulation from CT allows for an accurate and robust registration of the SSMs to intraoperative ultrasound images, with a success rate of $70 \%$ and a mean of $3.2 \mathrm{~mm}$. Additionally, no prior knowledge on the direction of the ultrasound probe is necessary.

3. The registration technique requires neither preprocessing nor segmentation in the US data. US segmentation is both time consuming and difficult to reproduce accurately.

4. Through a biomechanical model, the relative motion of the SSMs has been constrained to anatomically realistic orientations.

5. The proposed registration technique has been implemented on an nVidia GPU to reduce the computation time for data-intensive portions of the algorithm. Implementing the registration method on the GPU has been found to significantly decrease the registration time.

The registration algorithm has been validated on patient-based phantoms, specifically designed to retain the natural curvature of the spine. A special tissue mimicking material has been used to preserve realistic speckle information in the ultrasound data. The registration results shows that the algorithm can accurately register the shape and pose of the SSMs to ultrasound datasets, containing significant occlusion due to bony and soft-tissue interfaces. 


\subsection{Future Work}

While the registration method has been validated through limited phantom studies, it still requires further improvement to be ready for clinical applications. Future work will focus on improving the accuracy of the registration while reducing the registration run-time. The following are a few suggestions for future work:

- In order for this registration to be viable in interventional facilities, the registration time must be reduced to well below a minute. As a first step, to accomplish this goal, the most data intensive blocks of the registration framework have been implemented on the GPU. However, at the current stage, communication between different blocks in the registration algorithm is not optimized, requiring the CPU to act as a moderator. This imposes unnecessary transfer of data between host (CPU) and the device (GPU) at a hardware level. To further reduce the registration time, removing unnecessary communication between the CPU and the GPU is necessary.

- Another challenge in the registration procedure is the nature of the statistical shape models. The constructed SSMs do not retain any soft tissue information. Since in the registration process the only visible portions of the ultrasound images are the soft-tissue regions, adding it to the SSMs will provide a more realistic model. The soft-tissue is already visible in the patient CT data, however, due to extreme intensity variations, it is difficult to capture the soft-tissue information. A possible research direction is to use a B-spline intensity transform in addition to the B-spline deformable transform to incorporate soft-tissue into the SSM. 
- There is room for improvement of the biomechanical model used to represent the relative position and orientation of the SSMs. If this technique is to be extended to a larger number of vertebrae, a more detailed representation of the curvature of the spine is required. In the case of scoliotic patients, the biomechanical model is not adequate.

- While validation of the registration technique has been provided on a limited number of phantoms, to ensure the accuracy of the technique for clinical application, a through validation should be performed on cadaver data.

- Finally, since the main purpose of this US to CT registration technique is to assist in guiding the needle in spinal injections, an extensive user study should be performed and the accuracy of the guidance system evaluated. 


\section{Bibliography}

[1] Laurie Allan, Ute Richarz, Karen Simpson, and Robert Slappendel. Transdermal fentanyl versus sustained release oral morphine in strong-opioid naive patients with chronic low back pain. Spine, 30(22):2484-2490, 2005.

[2] Harris Allen, David Hubbard, and Sean Sullivan. The burden of pain on employee health and productivity at a major provider of business services. Journal of Occupational and Environmental Medicine, 47(7):658-670, 2005.

[3] Howard S. An. Percutaneous procedures in the lumbar spine, in Principles and Techniques of Spine Surgery. Lippincott Williams \& Wilkins, 1998.

[4] Dean C. Barratt, Carolyn S. K. Chan, Phillip J. Edwards, Graeme P. Penney, Mike Slomczykowski, Timothy J. Carter, and David J. Hawkes. Instantiation and Registration of Statistical Shape Models of the Femur and Pelvis Using 3D Ultrasound Imaging. Medical Image Analysis, 12(3):358-374, 2008.

[5] Dean C. Barratt, Graeme P. Penney, Carolyn S. K. Chan, Mike Slomczykowski, Timothy J. Carter, Philip J. Edwards, and David J. Hawkes. Self-calibrating 3dultrasound-based bone registration for minimally invasive orthopedic surgery. IEEE Transactions on Medical Imaging, 25(3):312-323, 2006. 
[6] Jonathan Boisvert, Farida Cheriet, Xavier Pennec, Hubert Labelle, and Nicholas Ayache. Geometric Variability of the Scoliotic Spine Using Statistics on Articulated Shape Models. IEEE Transactions on Biomedical Engineering, 27(4):557568, 2008.

[7] Amy E. Bonomi, Richard Shikiar, and Marcia West Legro. Quality-of-life assessment in acute, chronic, and cancer pain: Pain-related qol instruments used in assessing back pain. Journal of the American Pharmacists Association, 40:402416, 2000.

[8] Fred L. Bookstein. Size and shape spaces for landmark data in two dimensions. Statistical Science, 1(2):181-222, 1986.

[9] B. Brendel, S. Winter, A. Rick, M. Stockheim, and H. Ermert. Registration of 3D CT and ultrasound datasets of the spine using bone structures. Computer Aided Surgery, 7(3):146-155, 2002.

[10] Bernhard Brendel, Jennifer Siepermann, Susanne Winter, and Helmut Ermert. In vivo evaluation and in vitro accuracy measurements for an ultrasound-ct registration algorithm. International Congress Series, 1281:583 - 588, 2005. CARS 2005: Computer Assisted Radiology and Surgery.

[11] Jens Ivar Brox, Roger Srensen, Astrid Friis, ystein Nygaard, Aage Indahl, Anne Keller, Tor Ingebrigtsen, Hege R. Eriksen, Inger Holm, Anne Kathrine Kolleriise, Rolf Riise, and Olav Reikers. Randomized clinical trial of lumbar instrumented fusion and cognitive intervention and exercises in patients with chronic low back pain and disc degeneration. Spine, 28(17):1913-1921, 2003. 
[12] E.C.S. Chen, P. Mousavi, S. Gill, G. Fichtinger, and P. Abolmaesumi. Ultrasound-guided spine needle insertion. In SPIE Medical Imaging, page 762538, 2009.

[13] Thomas Kuiran Chen, Adrian D. Thurstonlow, Randy E. Ellis, and Purang Abolmaesumi. A real-time freehand ultrasound calibration system with automatic accuracy feedback and control. Ultrasound in Medicine 83 Biology, 1(1):79-93, 2009.

[14] T. F. Cootes, C. J. Taylor, D. H. Cooper, and J. Graham. Active shape models their training and application. computer vision and image understanding. Computer Vision and Image Understanding, 61(1):38-59, 1995.

[15] T.F Cootes, G.J. Edwards, and C.J. Taylor. Active appearance models. In Proceedings of European Conference on Computer Vision, 1998.

[16] Gregory D. Cramer and Susan A. Darby. Basic and Clinical Anatomy of the Spine, Spinal Cord, and ANS. C.V. Mosby, 2005.

[17] D. Crookes, K. Boyle, P. Miller, and C. Gillan. Gpu implementation of the affine transform for 3d image registration. In 13th International Conference on Machine Vision and Image Processing, 2009.

[18] Simon Dagenais, Jaime Caro, and Scott Haldeman. A systematic review of low back pain cost of illness studies in the united states and internationally. The Spine Journal, 8(1):8-20, 2008.

[19] Genevive Desroches, Carl-Eric Aubin, Daniel J. Sucato, and Charles-Hilaire Rivard. Simulation of an anterior spine instrumentation in adolescent idiopathic 
scoliosis using a flexible multi-body model. Medical and Biological Engineering and Computing, 45(8):759-768, 2007.

[20] Evelyn A. Firle, Wei Chena, and Stefan Wesarga. Registration of 3d u/s and ct images of the prostate. In Proceedings of the 16th International Congress and Exhibition Computer Assisted Radiology and Surgery, pages 527-532, 2002.

[21] Pezhman Foroughi, Danny Song, Gouthami Chintapani, Russel H. Taylor, and Gabor Fichtinger. Localization of Pelvic Anatomical Coordinate System Using US/Atlas Registration for Total Hip Replacement. In Medical Image Computing and Computer-Assisted Intervention, pages 871-878, 2008.

[22] S. Gill, P. Mousavi, G. Fichtinger, Elvis Chen, Jonathan Boisvert, David Pichora, and Purang Abolmaesumi. Biomechanically Constrained Groupwise US to CT Registration of the Lumbar Spine. In Medical Image Computing and ComputerAssisted Intervention, pages 803-810, 2009.

[23] Sean Gill, Purang Abolmaesumi, Gabor Fichtinger, Jonathan Boisvert, David Pichora, Dan Borshneck, and Parvin Mousavi. Biomechanically constrained groupwise ultrasound to ct registration of the lumbar spine. Medical Image Analysis, In Press, Accepted Manuscript, 2010.

[24] David G. Gobbi, Roch M. Comeau, and Terry M. Peters. Ultrasound/mri overlay with image warping for neurosurgery. In Medical Image Computing and Computer Assisted Interventions, 2000.

[25] Leiguang Gong, Jonathan Rohrer, Giridharan Iyengar, Brian Butler, and Alan Lumsden. Anatomical object recognition and labeling by atlas-based focused 
nonrigid registration and region-growing. In International Conference on Audio, Language and Image Processing, pages 1354-1358, 2008.

[26] R. H. Gong, J. Stewart, and P. Abolmaesumi. Reduction of Multi-Fragment Fractures of the Distal Radius Using Atlas-based 2D/3D Registration. In Proceedings of the SPIE, Medical Imaging 2009: Visualization, Image-Guided Procedures, and Modeling, volume 7261, pages 726137-1 - 726137-9, 2009.

[27] R. H. Gong, J. Stewart, and P. Abolmaesumi. A new representation of intensity atlas for GPUaccelerated instance generation. In to appear in the Proceedings of the IEEE Engineering in Medicine and Biology, 2010.

[28] Xiao Han, L.S. Hibbard, and V. Willcut. Gpu-accelerated, gradient-free mi deformable registration for atlas-based $\mathrm{mr}$ brain image segmentation. In IEEE Computer Society Conference on Computer Vision and Pattern Recognition Workshops, pages $141-148,20-252009$.

[29] Xiao Han, Lyndon S. Hibbard, and Virgil Willcut. Multi-atlas-based segmentation with local decision fusionapplication to cardiac and aortic segmentation in ct scans. IEEE Transactionms Medical Imaging, 28(7):1000-1010, 2009.

[30] Nikolaus Hansen, Sibylle D Mller, and Petros Koumoutsakos. Reducing the time complexity of the derandomized evolution strategy with covariance matrix adaptation. Evolutionary Computation, 11(1):1-18, 2003.

[31] Geremy Heitz, Torsten Rohlfing, and Calvin R. Maurer Jr. Statistical shape model generation using nonrigid deformation of a template mesh. In Proceedings of SPIE Medical Imaging, 2005. 
[32] Yipeng Hu, Hashim Uddin Ahmed, Clare Allen, Doug Pendse, Mahua Sahu, Mark Emberton, David Hawkes, and Dean Barratt. MR to Ultrasound Image Registration for Guiding Prostate Biopsy and Interventions. In Medical Image Computing and Computer-Assisted Intervention, pages 787-794, 2009.

[33] Xishi Huang, Nicholas A. Hill, Jing Ren, and Terry M. Peters. Rapid registration of multimodal images using a reduced number of voxels. In Proceedings of SPIE Medical Imaging, 2006.

[34] Xishi Huang, John Moore, Gerard Guiraudon, Douglas L. Jones, Daniel Bainbridge, Jing Ren, and Terry M. Peters. Dynamic 2d ultrasound and 3d ct image registration of the beating heart. IEEE Transactions on Medical Imaging, 28(8):1179-1189, 2009.

[35] V. Jurcak, J. Fripp, C. Engstrom, D. Walker, O. Salvado, S. Ourselin, and S. Crozier. Atlas Based Automated Segmentation of the Quadratus Lumborum Muscle Using Non-Rigid Registration on Magnetic Resonance Images of the Thoracolumbar Region. In 5th IEEE International Symposium on Biomedical Imaging: From Nano to Macro, pages 113-116, 2008.

[36] Andras Kelemen, Gabor Szekely, and Guido Gerig. Elastic model-based segmentation of 3-d neuroradiological data sets. IEEE Transactionms Medical Imaging, 18(10):828-839, 1999.

[37] Siavash Khallaghi, Parvin Mousavi, Ren Hui Gong, Sean Gill, Jonathan Boisvert, Gabor Fichtinger, David Pichora, Dan Borschneck, and Purang Abolmaesumi. Registration of a statistical shape model of the lumbar spine to $3 \mathrm{~d}$ ultrasound images. In Medical Image Computing and Computer Assisted Interventions, 2010. 
[38] A. P. King, K. S. Rhodem, Y. Ma, C. Yao, C. Yansen, R. Razavi, and G. P. Penney. Registering preprocedure volumetric images with intraprocedure 3-d ultrasound using an ultrasound imaging model. IEEE Transactions on Medical Imaging, 29(3):924-937, 2010.

[39] Rainer Klocke, Timothy Jenkinson, and David Glew. Sonographically guided caudal epidural steroid injections. Journal of Ultrasound in Medicine, 22(11):1229-1232, 2003.

[40] Oliver Kutter, Ramtin Shams, and Nassir Navaba. Visualization and gpuaccelerated simulation of medical ultrasound from ct images. Computer Methods and Programs in Biomedicine, 94(3):Computer Methods and Programs in Biomedicine, 2009.

[41] Oliver Kutter, Wolfgang Wein, and Nassir Navab. Multi-Modal Registration Based Ultrasound Mosaicing. In Medical Image Computing and ComputerAssisted Intervention, pages 763-770, 2009.

[42] Thomas Lange, Sebastian Eulenstein, Michael Hnerbein, Hans Lamecker, and Peter-Michael Schlag. Augmenting intraoperative 3d ultrasound with preoperative models for navigation in liver surgery. In Medical Image Computing Computer Assisted Intervention, 2004.

[43] Koen Van Leemput. Encoding probabilistic brain atlases using bayesian inference. IEEE Transactions on Medical Imaging, 28(6):822-837, 2009. 
[44] Aaron E. Lefohn, Joshua E. Cates, and Ross T. Whitaker. Interactive, gpu-based level sets for 3d segmentation. In Medical Image Computing and ComputerAssisted Intervention, 2003.

[45] Antoine Leroy, Pierre Mozer, Yohan Payan, and Jocelyne Troccaz. Rigid registration of freehand 3d ultrasound and ct-scan kidney images. In Medical Image Computing and Computer-Assisted Intervention, 2004.

[46] Environics Research Group Limited. Survey of canadian adults: Back pain. Toronto, Canada, 2003.

[47] Alan B. Lippitt. The facet joint and its role in spine pain: Management with facet joint injections. Spine, 9(7):746-750, 1984.

[48] Cristian Lorenz and Nils Krahnstver. Generation of point-based 3d statistical shape models for anatomical objects. Computer Vision and Image Understanding, 77(2):175-191, 2000.

[49] Ernest L Madsen, Maritza A Hobson, Hairong Shi, Tomy Varghese, and Gary R Frank. Tissue-mimicking agar/gelatin materials for use in heterogeneous elastography phantoms. Physics in Medicine and Biology, 50(Issue 23):5597-5618, 2005.

[50] Nikolaos Maniadakisa and Alastair Gray. The economic burden of back pain in the uk. Pain, 84(1):95-103, 2000.

[51] Robert Cornelius Marks, Thomas Houstonb, and Terry Thulbournea. Facet joint injection and facet nerve block: a randomised comparison in 86 patients with chronic low back pain. Pain, 49(3):325-328, 1992. 
[52] L. Mei, M. Figi, D. Rueckert, A. Darzi, and P. Edwards. Sample sufficiency and number of modes to retain in statistical shape modeling. In Medical Image Computing and Computer Assisted Interventions, 2008.

[53] Sofia K. Michopoulo, Lena Costaridou, Elias Panagiotopulous, Robert Speller, George Panayiotakis, and Andrew Todd-Pokropek. Atlas-Based Segmentation of Degenerated Lumbar Interverbal Discs from MR Images of the Spine. IEEE Transactions on Biomedical Engineering, 56(9):2225-2231, 2009.

[54] Mehdi Hejazi Moghari and Purang Abolmaesumi. Point-based rigid-body registration using an unscented kalman filter. IEEE Transactions on Medical Imaging, 26(12):1708-1728, 2007.

[55] John Moore, Colin Clarke, Daniel Bainbridge, Chris Wedlake, Andrew Wiles, Danielle Pace, and Terry Peters. Image Guidance for Spinal Facet Injections Using Tracked Ultrasound. In Medical Image Computing and Computer-Assisted Intervention, pages 516-523, 2009.

[56] Reed F. Murtagh. Computed tomography and fluoroscopy guided anesthesia and steroid injection in facet syndrome. Spine, 13(6):686-689, 1988.

[57] P. Muyan-Ozcelik, J.D. Owens, Junyi Xia, and S.S. Samant. Fast deformable registration on the gpu: A cuda implementation of demons. In International Conference on Computational Sciences and Its Applications, pages 223-233, june 2008.

[58] R. Narayanan, D. Shen, C. Davatzikos, D. E. Crawford, A. Barqawi, P. N. Werahera, D. Kumar, and J. S. Suri. Application and validation of registration 
framework for real-time atlas guided biopsy. In 5th IEEE International Symposium on Biomedical Imaging: From Nano to Macro, 2008.

[59] Arunachalam Narayanaswamy, Saritha Dwarakapuram, Christopher S. Bjornsson, Barbara M. Cutler, William Shain, and Badrinath Roysam. Robust adaptive 3-d segmentation of vessel laminae from fluorescence confocal microscope images and parallel gpu implementation. IEEE Transactions on Medical Imaging, 29(3):583-597, 2010.

[60] G.P. Penney, J.M. Blackall, M.S. Hamady, T. Sabharwal, A. Adam, and D.J. Hawkes. Registration of freehand 3d ultrasound and magnetic resonance liver images. Medical Image Analysis, 8(1):81-91, 2004.

[61] Josien P. W. Pluim, J. B. Antoine Maintz, and Max A. Viergever. Image registration by maximization of combined mutual information and gradient information. IEEE Transactions on Medical Imaging, 19(8):809-814, 2000.

[62] B.C. Porter, D.J. Rubens, J.G. Strang, J. Smith, S. Totterman, and K.J. Parker. Three-dimensional registration and fusion of ultrasound and mri using major vessels as fiducial markers. IEEE Transactionms Medical Imaging, 20(4):354$359,2001$.

[63] P. Prithvi Raj, Leland Lou, Serdar Erdine, Peter S. Staats, and Steven D. Waldman. Radiographic Imaging for Regional Anaesthesia and Pain Management. Churchill Livingstone, Elsevier Science, Philadelphia, 2003.

[64] Kumar T. Rajamani, Martin A. Styner, Haydar Talib, Guoyan Zheng, Lutz P. Nolte a, and Miguel A. Gonzalez Ballester. Statistical deformable bone models 
for robust 3d surface extrapolation from sparse data. Medical Image Analysis, 11(2):99-109, 2007.

[65] Alexis Roche, Xavier Pennec, Grgoire Malandain, and Nicholas Ayache. Rigid registration of $3 \mathrm{~d}$ ultrasound with $\mathrm{mr}$ images: A new approach combining intensity and gradient information. IEEE Transactions on Medical Imaging, 20(10):1038-1048, 2001.

[66] Ofri Sadowsky, Jonathan D. Cohen, and Russel H. Taylor. Rendering Tetrahedral Meshes with Higher-Order Attenuation Functions for Digital Radiograph Reconstruction. In IEEE Visualization, pages 303-310, 2005.

[67] R. Shams, P. Sadeghi, R. Kennedy, and R. Hartley. A survey of medical image registration on multicore and the gpu. IEEE Signal Processing Magazine, 27(2):50 -60, march 2010.

[68] Wei Shao, Ruoyun Wu, Keck Voon Ling, Choon Hua Thng, Henry Sun Sien Ho, Christopher Wai Sam Cheng, , and Wan Sing Ng. Evaluation on similarity measures of a surface-to-image registration technique for ultrasound images. In Medical Image Computing Computer Assisted Intervention, 2006.

[69] Walter F. Stewart, Judith A. Ricci, Elsbeth Chee, David Morganstein, and Richard Lipton. Lost productive time and cost due to common pain conditions in the us workforce. The Journal of the American Medical Association, 290(18):2443-2454., 2003.

[70] Walter F. Stewart, Judith A. Ricci, Elsbeth Chee, David Morganstein, and Richard Lipton. Lost productive time and cost due to common pain conditions 
in the us workforce. Journal of the American Medical Association, 290(18):2443$2454,2003$.

[71] Haydar Talib, Kumar Rajamani, Jens Kowal, Lutz-Peter Nolte, Martin Styner, and Miguel A. Gonzalez Ballester. A Comparison Study Assessing the Fesibility of Ultrasound-Initialized Deformable Bone Models. Computer Aided Surgery, 10(5/6):293-299, 2005.

[72] Thomas S.Y. Tang and Randy E. Ellis. 2D/3D Deformable Registration Using a Hybrid Atlas. In Medical Image Computing and Computer-Assisted Intervention, pages 223-230, 2005.

[73] Tauseef ur Rehman, Eldad Haber, Gallagher Pryor, John Melonakos, and Allen Tannenbauma. 3d nonrigid registration via optimal mass transport on the gpu. Medical Image Analysis, 13(6):931-940, 2009.

[74] M. J. Watson, S. Evans, and J. M. Thorp. Could ultrasonography be used by an anaesthetist to identify a specified lumbar interspace before spinal anaesthesia? British Journal of Anaesthesia, 90(4):509-511, 2003.

[75] R.W. Watts. A five-year prospective analysis of the efficacy, safety and morbidity of epidural anaesthesia performed by a general practitioner anaesthetist in an isolated rural hospital. Anaesth Intensive Care, 20(3):348-353, 1992.

[76] Wolfgang Wein, Shelby Brunke, Ali Khamene, Matthew R. Callstrom, and Nassir Navab. Automatic CT-ultrasound registration for diagnostic imaging and imageguided intervention. Medical Image Analysis, 12(5):577-585, 2008. 
[77] Wolfgang Wein, Barbara Roper, and Nassir Navab. Automatic registration and fusion of ultrasound with ct for radiotherapy. In Medical Image Computing Computer Assisted Intervention, 2005.

[78] Arthur H. White, Richard Derby, and Gar Wynne. Epidural injections for the diagnosis and treatment of low-back pain. Spine, 5(1):78-86, 1980.

[79] S. Winter, B. Brendel, I. Pechlivanis, K. Schmieder, and C. Igel. Registration of $\mathrm{CT}$ and intraoperative 3-D ultrasound images of the spine using evolutionary and gradient-based methods. IEEE Transactions on Evolutionary Computation, 12(3):284-296, 2008.

[80] Jeong Won-Ki, J. Beyer, M. Hadwiger, A. Vazquez, H. Pfister, and R.T. Whitaker. Scalable and interactive segmentation and visualization of neural processes in em datasets. IEEE Transactions on Visualization and Computer Graphics, 15(6):1505 - 1514, 2009.

[81] Michael Zenz, Michael Strumpf, and Michael Tryba. Long-term oral opioid therapy in patients with chronic nonmalignant pain. Journal of Pain and Symptom Management, 7(2):69-77, 1992.

[82] Yongxin Zhou and Jing Bai. Multiple abdominal organ segmentation: An atlasbased fuzzy connectedness approach. IEEE Transactions on Information Technology in Biomedicine, 11(3):348-352, 2007. 


\section{Appendix A}

\section{Parallel Implementation on the GPU}

In this appendix, a brief explanation of the GPU accelerated instance generation and ultrasound simulation is given. As it was show in section 4.3.5, a huge speed up is gained using parallel implementation on the GPU.

\section{GPU Accelerated Instance Generation}

Figure A.1 illustrates the parallel instance generation algorithm. Given the atlas weights, a linear combination of eigenvectors is achieved through equation 4.1 and set as the Bspline grid node vectors. The atlas template and grid node vectors are uploaded from the global memory to the device memory and stored in CUDA3D-arrays. A 3D-texture is also bound to each array, enabling the algorithm to read and manipulate data in the 3D-arrays. We have implemented the instantiation in two steps. The first kernel, interpolates the B-spline grid nodes to produce the deformation field at each voxel. This is done by placing a $2 \mathrm{D}$ grid that can span 


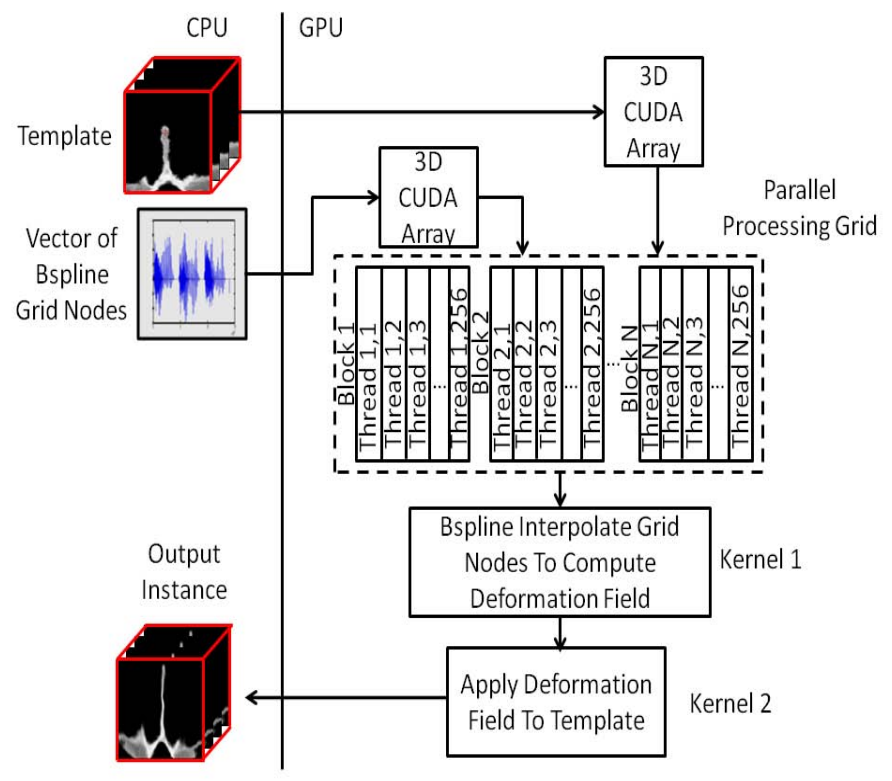

Figure A.1: Parallel implementation of instance generation in GPU.

each slice and then processing one slice at a time to produce the deformation vector. Subsequently, the second kernel transforms each voxel from the index representation to the physical coordinate system, applies the deformation vector and transforms it back to the index representation. In the case of unassigned voxels in the final volume, the empty voxel is filled with a default value. Finally the deformed volume is downloaded to global memory.

\section{GPU Accelerated Ultrasound Simulation}

Figure A.2 illustrates the parallel implementation of ultrasound simulation on the GPU. First the input CT volume is uploaded to the device memory and stored as a CUDA array. Then, a 2D grid is placed on top of the CT volume parallel to the coronal plane. the ultrasound is simulated in two steps: The first CUDA kernel computes 
the ultrasound reflection image, following equations 4.34 .7 , each ultrasound ray is assigned one CUDA-thread, traveling down the CT volume. The second kernel, computes the mapped CT image using equation 4.8. The two images are fused together using equation 4.9 to produce the simulated ultrasound. The result of the ultrasound simulation is then downloaded to the global memory.

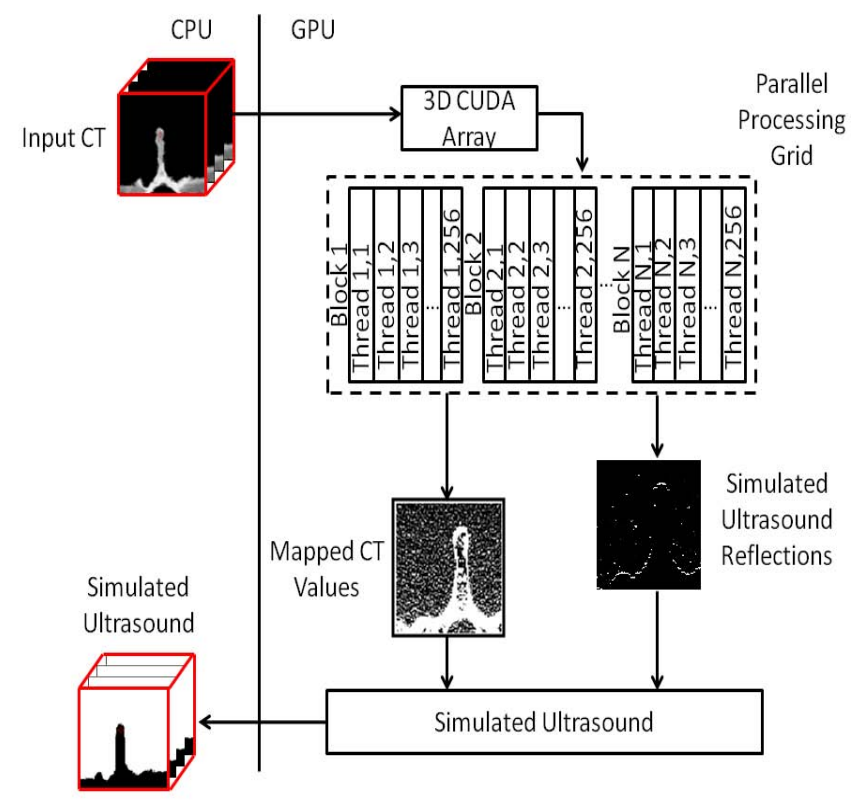

Figure A.2: Parallel implementation of ultrasound simulation on the GPU. 